\title{
Holographic Rényi entropy for CFT with $W$ symmetry
}

\author{
Bin Chen, ${ }^{a, b, c}$ Jiang Long ${ }^{a}$ and Jia-ju Zhang ${ }^{a}$ \\ ${ }^{a}$ Department of Physics and State Key Laboratory of Nuclear Physics and Technology, \\ Peking University, 5 Yiheyuan Rd, Beijing 100871, P.R. China \\ ${ }^{b}$ Collaborative Innovation Center of Quantum Matter, \\ 5 Yiheyuan Rd, Beijing 100871, P.R. China \\ ${ }^{c}$ Center for High Energy Physics, Peking University, \\ 5 Yiheyuan Rd, Beijing 100871, P.R. China \\ E-mail: bchen01@pku.edu.cn, 1j301@pku.edu.cn, jjzhang@pku.edu.cn
}

ABSTRACT: In this paper we investigate the holographic Rényi entropy of two disjoint intervals on complex plane with small cross ratio $x$ for conformal field theory with $W$ symmetry in the ground state, which could be dual to a higher spin $\mathrm{AdS}_{3}$ gravity. We focus on the cases of $W_{3}$ and $W_{4}$ symmetries. In order to see the nontrivial contributions from the $W$ fields, we calculate the Rényi entropy in the expansion of $x$ to order $x^{8}$ in both the gravity and the CFT sides. In the gravity side the classical contributions to the entanglement entropy is still given by the Ryu-Takayanagi area formula under the reasonable assumption, while the 1-loop quantum corrections have to take into account of the contributions not only from massless gravitons, but also from massless higher spin fields. In the CFT side we still use the operator product expansion of twist operators in the small interval limit, but now we need to consider the quasiprimary fields constructed from $W$ fields, besides the ones from Virasoro Verma module. In the large central charge limit, we obtain the classical, 1-loop, 2-loop, and 3-loop parts of the Rényi entropy. The classical and 1-loop results in the gravity and the CFT sides are in exact match. This confirms the higher spin gravity/CFT correspondence, and also supports the holographic computation of Rényi entanglement entropy, including the quantum correction, in both the $\mathrm{AdS}_{3}$ gravity and the higher spin $\mathrm{AdS}_{3}$ gravity.

Keywords: AdS-CFT Correspondence, Conformal and W Symmetry, Holography and condensed matter physics (AdS/CMT)

ARXIV EPRINT: 1312.5510 


\section{Contents}

1 Introduction 1

2 Holographic Rényi entropy 4

2.1 The classical part 4

2.2 The 1-loop correction 5

2.2.1 Graviton 6

2.2.2 Spin-3 field 6

2.2.3 Spin-4 field 6

3 Rényi entropy in the CFT side $\quad 6$

$\begin{array}{lll}3.1 & \text { OPE of twist operators } & 7\end{array}$

3.2 Ordinary CFT 9

$\begin{array}{lll}3.3 & \text { CFT with } W(2,3) \text { symmetry } & 10\end{array}$

$\begin{array}{lll}3.4 & \text { CFT with } W(2,4) \text { symmetry } & 11\end{array}$

3.5 CFT with $W(2,3,4)$ symmetry 11

4 Conclusion and discussion $\quad 12$

$\begin{array}{ll}\text { A Counting of quasiprimary operators } & 13\end{array}$

$\begin{array}{ll}\text { B The coefficients } d_{K} & 19\end{array}$

$\begin{array}{ll}\text { C Some details in subsection } 3.2 & 22\end{array}$

$\begin{array}{ll}\text { D One short interval on cylinder } & 23\end{array}$

E Some useful summation formulas $\quad 24$

\section{Introduction}

In this paper we continue the study of the short interval expansion of Rényi entropy of two-dimensional (2D) conformal field theory (CFT) initiated in [1-3]. Let us first review the basic definition of Rényi entropy. The entanglement Rényi entropy is an important notion of quantum systems, and it is defined as follows $[4,5]$. One can divide a system into two parts, say $A$ and its complement $B$. From the density matrix $\rho$ of the whole system one may obtain the reduced density matrix of $A$ by tracing over the degrees of freedom of $B$, i.e. $\rho_{A}=\operatorname{Tr}_{B} \rho$. Then the entanglement entropy of $A$ and $B$ is defined as

$$
S_{A}=-\operatorname{Tr}_{A} \rho_{A} \log \rho_{A} .
$$


More generally one can define the Rényi entropy of $A$ and $B$ as

$$
S_{A}^{(n)}=-\frac{1}{n-1} \log \operatorname{Tr}_{A} \rho_{A}^{n}
$$

The entanglement entropy and the Rényi entropy are related by $S_{A}=\lim _{n \rightarrow 1} S_{A}^{(n)}$. Moreover one may choose two subsystems $A$ and $B$ which are not necessarily complementary to each other, and define the Rényi mutual information of $A$ and $B$

$$
I_{A, B}^{(n)}=S_{A}^{(n)}+S_{B}^{(n)}-S_{A \cup B}^{(n)} .
$$

The mutual information $I_{A, B}^{(1)}$ encodes the entanglement between $A$ and $B$.

The standard way of computing the Rényi entropy is the replica trick [6], but it is usually hard to operate for a general system. Some computations in 2D CFT and higher dimensional free field theory could be found in [1, 2, 7-14]. For a CFT with a gravity dual one may use the AdS/CFT correspondence [15-17] to do a simpler holographic computation in the bulk gravity. For the pure AdS gravity, it was conjectured by Ryu and Takayanagi that the leading contribution of entanglement entropy is captured by the area of a minimal surface in the bulk with asymptotic boundary ending on $A$ [18-21]. This socalled RT area law is reminiscent of the black hole entropy, and therefore since its proposal there have been many attempts to derive this law in the gravity [22-25]. Very recently, the RT area law of holographic entanglement entropy has been proved in [26] by taking it as a generalized gravitational entropy. However it is fair to say that for dimension $D \geq 3$ $\mathrm{CFT}$, it is not certain if the holographic entanglement entropy is exactly the entanglement entropy in the field theory, as there is short of direct computations in the field theory. Nevertheless for 2D CFT, the situation is much clearer.

In 2D CFT, due to its infinite dimensional conformal symmetries, the direct field computation is feasible. One could insert the twist operators to impose the nontrivial boundary conditions in applying the replica trick [7, 9]. As a result, the partition function on a higher-genus Riemann surface could be recast into the correlators of twist fields on a complex plane in an orbifold CFT, which arises from the $Z_{n}$ replica symmetry. For a 2D CFT on complex plane the Rényi entropy for one interval with length $\ell$ is universal and only depends on the central charge [7]

$$
S_{n}=\frac{c}{6}\left(1+\frac{1}{n}\right) \log \frac{\ell}{\epsilon}
$$

with $\epsilon$ being the UV cutoff. For the entanglement entropy $S_{1}$, it has been reproduced by the holographic computation in $[18,19]$. For the general $S_{n}, n>1$, they have been reproduced successfully in [27] from the Euclidean action of corresponding gravitational configurations. For multi-interval case, the correctness of RT law and its agreement with CFT result have been proved in [27] and [28], respectively.

In $\mathrm{AdS}_{3} / \mathrm{CFT}_{2}$ correspondence, the central charge of CFT is inversely proportional to the bulk Newton constant [29]

$$
c=\frac{3 l}{2 G_{N}^{(3)}}
$$


where $l$ is the radius of $\mathrm{AdS}_{3}$ spacetime and $G_{N}^{(3)}$ is the coupling constant. The RT area law actually captures the contribution proportional to $c$. In the large central charge limit, this is the leading contributions in CFT. For the multi-interval cases, there are subleading contributions, which could be essential in discussing the issues like mutual information. From AdS/CFT correspondence, these subleading contributions should be counted as the quantum corrections to gravitational action $[1,30,31]$. In particular, the quantum correction to the holographic Rényi entropy has been studied in [30]. In the small interval limit with a small cross ratio $x$ on complex plane, the quantum 1-loop correction of graviton to the Rényi mutual information has been calculated to order $x^{8}$. According to the AdS/CFT correspondence, the graviton in the bulk corresponds to the stress tensor in the CFT side. To account the graviton correction, it is only necessary to consider the Virasoro Verma module in CFT. Actually from the study of quantum gravity in $\mathrm{AdS}_{3}$, it has been known that the pure gravity partition function could be reproduced from the Virasoro Verma module [32]. In [3] by studying the OPE of twist operators at small interval limit, the Rényi mutual information of two interval has been computed in the CFT side. The quantum 1-loop correction of graviton to the Rényi mutual information in [30] has been confirmed to order $x^{6}$. This strongly support the holographic computation of Rényi entropy beyond the classical level.

In this paper we continue the work initiated in [3]. In this work we would like to consider the CFT with $W$ symmetry in its ground state. In this case, the dual gravity could be a higher spin (HS) $\mathrm{AdS}_{3}$ gravity theory [33, 34]. Therefore our investigations may not only shed light on the holographic Rényi entropy with matter coupling, but also allows us to understand the HS/CFT correspondence from a new angle. We focus on the Rényi mutual information of two disjoint intervals with small cross ratio. In order to see the contributions from $W$ fields with conformal weights 3 and 4 clearly, we need to find the contributions up to order $x^{8}$. Using the method in [30], we calculate the quantum 1-loop contributions of spin-3 and spin-4 fields to the Rényi mutual information of the two interval case to order $x^{8}$. On the gravity side, under the assumption the classical configurations are still the handlebody geometries constructed in [27], we consider the 1-loop fluctuations around these configurations. One feature of the calculation is that the contributions of different spin fields are additive, and so we can calculate them separately. On the CFT side, we firstly consider only the contributions from the Virasoro Verma module and verify the results in [30] to order $x^{8}$. Then we consider the extra contributions from $W$ fields in the CFT side. The leading contribution of the $W_{m}$ field to the Rényi mutual information in the CFT is of order $x^{2 m}$. This can be got easily, and matches the bulk result. The subleading contributions are more difficult to get. Different from the calculation in the gravity side, in the CFT side the contributions of $W$ fields cannot be considered separately, as they are involved with the stress tensor. We verify that the contributions of $W_{3}$ field and/or $W_{4}$ field to order $x^{8}$ match exactly with the gravity results as well. This shows that the holographic prescription of computing the entanglement Rényi entropy for the ground state CFT not only applies to an ordinary CFT but also to CFTs with $W$ symmetries.

The remaining of the paper is arranged as follows. In section 2 we calculate the classical Rényi entropy as well as the 1-loop corrections of spin-3 and spin-4 fluctuations to order $x^{8}$ in the gravity side. In section 3 we confirm these results in the CFT side using the short 
interval expansion of the Rényi entropy. We end with conclusion and discussion in section 4 . Some details of the computation in section 3 are presented in the appendixes A, B, and C. The appendix $\mathrm{D}$ is a byproduct of the paper and it is about the case of one short interval on cylinder. Some useful summation formulas are collected in appendix E.

Note added. The same day this paper appeared in arXiv, there appeared another paper [35] which has some overlaps with our work.

\section{Holographic Rényi entropy}

In this section we calculate the classical and 1-loop parts of the holographic Rényi entropy for two intervals with small cross ratio in CFT with $W$ symmetry. As we are considering the entanglement entropy in the vacuum state of CFT, we focus on the $\mathrm{AdS}_{3}$ vacuum. The gravitational configurations are the same as the ones worked out in $[27,30]$. Therefore, we assume that the classical part coming from bulk gravitational action is invariant. ${ }^{1}$ But we have not only the massless boundary gravitons but also massless higher spin fluctuations, all of which contribute to 1-loop quantum correction. As the massless field with higher spin $m$ corresponds to a pair of holomorphic and antiholomorphic operators with conformal weight $m$, their contributions to the Rényi entropy is of order $x^{2 m}$. In order to read the nontrivial information, we need to do higher order expansion of $x$. In this work, we manage to work out the contributions to order $x^{8}$, which allows us to discuss the higher spin gravity to spin-4 field.

The higher spin gravity has been under active study in the past few years. For the pure spin-3 $\mathrm{AdS}_{3}$ gravity, it was proposed in [33] that its action could be written in terms of Chern-Simons form with gauge group $\mathrm{SL}(3, R)$. Moreover, as its asymptotic symmetry group has $W_{3}$ symmetry, it was conjectured that the spin-3 $\mathrm{AdS}_{3}$ gravity could be holographically dual to a conformal field theory with $W_{3}$ symmetry but the same central charge. More generally, the Chern-Simons gravity could be defined with other Lie groups, describing the interaction of higher spin fields with gravity. When the gauge group is $\mathrm{SL}(4, R)$, it describe both the spin- 4 and spin-3 fields interacting with the gravity, which is dual to a CFT with $W(2,3,4)$ symmetry. But one may obtain only the spin-4 field interacting with the gravity by choosing the gauge group to be $\mathrm{SO}(5)$ or $\mathrm{Sp}(4)$ [36]. This truncated spin- 4 gravity is conjectured to be dual to a CFT with $W(2,4)$ symmetry. In all these cases, the dual CFT has the same central charge as the one for pure $\mathrm{AdS}_{3}$ gravity, so that all the higher spin fields could be set to vanish without spoiling the underlying correspondence. In other words, all the classical gravitational configurations in [30] are still the classical solutions of higher spin gravity and moreover their bulk classical actions would not be changed by the presence of higher spin fields.

\subsection{The classical part}

The classical Rényi entropy for a 2D CFT with large central charge could be calculated using the method proposed in $[27,28]$. It reduces to the monodromy problem of an ordinary differential equation. In [30] the method was used to calculate the small cross ratio

\footnotetext{
${ }^{1}$ For more careful justification of this assumption in higher spin gravity, see [35].
} 
expansion of the classical Rényi entropy for the two intervals case. Here we adopt the same prescription but only give the outline without much details. One could find the details of the calculation in [30].

We choose the subregion $A=(-\infty,-1] \cup[-y, y] \cup[1,+\infty)$ with $y$ being small, and have the cross ratio

$$
x=\frac{4 y}{(1+y)^{2}},
$$

which is small too. The classical Rényi entropy could be obtained using

$$
\frac{\partial S_{n}^{c l}}{\partial y}=-\frac{c n}{3(n-1)} \gamma_{3}
$$

with

$$
\begin{aligned}
\gamma_{3}= & -\frac{n^{2}-1}{2 n^{2} y}+\frac{2\left(n^{2}-1\right)^{2} y}{3 n^{4}}+\frac{2\left(n^{2}-1\right)^{2}\left(49 n^{4}-2 n^{2}-11\right) y^{3}}{135 n^{8}} \\
& +\frac{2\left(n^{2}-1\right)^{2}\left(3211 n^{8}-172 n^{6}-1056 n^{4}-172 n^{2}+376\right) y^{5}}{8505 n^{12}} \\
& +\frac{2\left(n^{2}-1\right)^{2} y^{7}}{1913625 n^{16}}\left(740087 n^{12}-44106 n^{10}-290847 n^{8}-78748 n^{6}+149973 n^{4}\right. \\
& \left.+67854 n^{2}-58213\right)+O\left(y^{9}\right) .
\end{aligned}
$$

And then we can get the classical Rényi mutual information

$$
\begin{aligned}
I_{n}^{c l}= & \frac{c(n+1)}{6 n} \log y-S_{n}^{c l} \\
= & \frac{c(n-1)(n+1)^{2} y^{2}}{9 n^{3}}+\frac{c(n-1)(n+1)^{2}\left(49 n^{4}-2 n^{2}-11\right) y^{4}}{810 n^{7}} \\
& +\frac{c(n-1)(n+1)^{2}\left(3211 n^{8}-172 n^{6}-1056 n^{4}-172 n^{2}+376\right) y^{6}}{76545 n^{11}} \\
& +\frac{c(n-1)(n+1)^{2} y^{8}}{22963500 n^{15}}\left(740087 n^{12}-44106 n^{10}-290847 n^{8}-78748 n^{6}+149973 n^{4}\right. \\
& \left.+67854 n^{2}-58213\right)+O\left(y^{10}\right) .
\end{aligned}
$$

Obviously, when $n=1$, the classical part of the mutual information in this case is vanishing, and this matches the RT area formula [1].

\subsection{The 1-loop correction}

The method of calculating the 1-loop correction of the Rényi entropy was given in [30], and it is directly related to the computation of the partition function $[37,38]$. The relation is that

$$
S_{n}^{1-\mathrm{loop}}=-\frac{1}{n-1}\left(\log Z_{n}^{1-\mathrm{loop}}-n \log Z_{1}^{1-\mathrm{loop}}\right) .
$$

For the two intervals on complex plane case, we have $Z_{1}^{1-\text { loop }}=1$ and the second term of the right hand side of the above equation can be omitted. 


\subsubsection{Graviton}

The partition function depends on the matter content of the gravity as well as the spacetime configuration. When the spacetime is the quotient of global $\mathrm{AdS}_{3}$ by a Schottky group $\Gamma$, the 1-loop partition function for the graviton is [38]

$$
Z^{1 \text {-loop }}=\prod_{\gamma \in \mathcal{P}} \prod_{m=2}^{\infty} \frac{1}{\left|1-q_{\gamma}^{m}\right|},
$$

with $\mathcal{P}$ being a set of representatives of the primitive conjugacy classes of $\Gamma$. Using this method, the small cross ratio $x$ expansion of the 1-loop correction of Rényi mutual information to order $x^{8}$ has been given in [30], and it has been confirmed to order $x^{6}$ from CFT computation by considering only the contributions from the Virasoro Verma module [3].

\subsubsection{Spin-3 field}

In the presence of higher spin fields, the 1-loop partition function becomes [39]

$$
Z^{1-\text { loop }}=\prod_{\gamma \in \mathcal{P}} \prod_{s} \prod_{m=s}^{\infty} \frac{1}{\left|1-q_{\gamma}^{m}\right|} .
$$

Here the product over $s$ is with respect to the spins of massless fields. For $s=2$ it reduces to the graviton case, and for $s \geq 3$ it corresponds to the higher spin fields. One feature of this formula is that the contributions of the fields with different spins to the Rényi entropy could be separated. Therefore, the contribution from the gravitons is the same as the one got in [30]. For the spin-3 field, the contribution to the Rényi mutual information is

$$
\begin{aligned}
I_{n, \text { spin-3 }}^{1-\text { loop }}= & \frac{n}{n-1}\left(\frac{f_{6} x^{6}}{4096 n^{12}}+\frac{3\left(f_{7}+\left(n^{2}-1\right) f_{6}\right) x^{7}}{4096 n^{14}}\right. \\
& \left.+\frac{\left(273 f_{8}+504\left(n^{2}-1\right) f_{7}+2\left(137 n^{4}-250 n^{2}+113\right) f_{6}\right) x^{8}}{196608 n^{16}}+O(x)^{9}\right) .
\end{aligned}
$$

The functions $f_{m}$ 's are defined in (E.1), and the explicit form of $I_{n, \text { spin-3 }}^{1-\text { loop }}$ could be got easily using (E.2). Note that the singular behavior in $I_{n, \text { spin-3 }}^{1-\text { loop }}$ at $n=1$ is superficial. Actually, the $(n-1)$ factor in the denominator is cancelled by the $\left(n^{2}-1\right)$ factor in the $f_{m}$ 's.

\subsubsection{Spin-4 field}

For the spin- 4 field, the contribution is

$$
I_{n, \mathrm{spin}-4}^{1-\mathrm{loop}}=\frac{n}{n-1}\left(\frac{f_{8} x^{8}}{65536 n^{16}}+O(x)^{9}\right) .
$$

\section{Rényi entropy in the CFT side}

In this section we compute the short interval expansion of the Rényi entropy in the CFT side, and we mainly focus on the case of two intervals on complex plane with small cross ratio. As we are considering the CFT with $W$ symmetry, we must take into account quasi-primary fields constructed from the primary $W$ operators. 


\subsection{OPE of twist operators}

The short interval expansion of Rényi entropy has been discussed intensively in [1-3], and here we only outline the key points.

A systematic way of computing the $n$-th Rényi entropy of $N$ disjoint intervals for a general 2D CFT on complex plane was proposed in [7]. The replica trick requires us to compute the partition function of the CFT on the Riemann surface $R_{n, N}$ of genus $(n-1)(N-1)$, which originates from the sewing of $n$ copies of the complex plane with branch cuts. Alternatively, one may work with $n$ copies of the original CFT on a complex plane but with nontrivial boundary conditions relating the fields of different replicas at the branch points. Note that In the first picture there is one copy of the original CFT on $\mathcal{R}_{n, N}$ which is an orbifold CFT, and in the second picture there is one copy of the complex plane but the CFT consists of $n$ copies of the original CFT and so will be denoted by $C F T^{n}$. In the second picture the boundary conditions could be accounted by inserting the twist operators $\sigma(z, \bar{z}), \tilde{\sigma}(z, \bar{z})$ at the branch points which are the boundaries of the intervals. The twist operators are primary operators with conformal weights

$$
h=\bar{h}=\frac{c}{24}\left(n-\frac{1}{n}\right),
$$

with $c$ being the central charge of the original CFT.

In the first picture we denote a local operator as $\phi\left(z_{j}, \bar{z}_{j}\right)$ with $z_{j}$ being the coordinate of $j$-th copy of the plane, and in the second picture we denote a local operator as $\phi_{j}(z, \bar{z})$ with $\phi_{j}$ being an operator of the $j$-th copy of the original CFT and $z$ being the coordinate of the complex plane. The operators in the two pictures can be converted freely, and sometimes we just mix the two kinds of symbols for simplicity. For example, we take

$$
T\left(z_{j_{1}}, \bar{z}_{j_{1}}\right) T\left(z_{j_{2}}, \bar{z}_{j_{2}}\right) \equiv T_{j_{1}}(z, \bar{z}) T_{j_{2}}(z, \bar{z}), \quad j_{1} \neq j_{2}
$$

with the left side being an operator in the first picture, which is nonlocal, and the right side being an operator in the second picture, which is local, but the operators are taken to be different.

If we choose $A=\left[z_{1}, z_{2}\right] \cup \cdots \cup\left[z_{2 N-1}, z_{2 N}\right]$, we have

$$
\operatorname{Tr} \rho_{A}^{n}=\left\langle\sigma\left(z_{2 N}, \bar{z}_{2 N}\right) \tilde{\sigma}\left(z_{2 N-1}, \bar{z}_{2 N-1}\right) \cdots \sigma\left(z_{2}, \bar{z}_{2}\right) \tilde{\sigma}\left(z_{1}, \bar{z}_{1}\right)\right\rangle_{C} .
$$

When $N=1$ and $A=[0, \ell]$, we have

$$
\operatorname{Tr} \rho_{A}^{n}=\langle\sigma(\ell, \ell) \tilde{\sigma}(0,0)\rangle_{C}=c_{n} \ell^{-\frac{c}{6}\left(n-\frac{1}{n}\right)},
$$

with $c_{n}$ being a constant related to the normalization of the twist operators. Then the Rényi entropy for one interval could be found [7]

$$
S_{n}=\frac{c}{6}\left(1+\frac{1}{n}\right) \log \frac{\ell}{\epsilon}
$$

with $\epsilon$ being the UV cutoff. 
We denote the linearly independent quasiprimary operators in $C F T^{n}$ as $\Phi_{K}(z, \bar{z})$ with the conformal wights $h_{K}$ and $\bar{h}_{K}$. Moreover we orthogonalize these operators as

$$
\left\langle\Phi_{K}(z, \bar{z}) \Phi_{L}(w, \bar{w})\right\rangle_{C}=\frac{\delta_{K L} \alpha_{K}}{(z-w)^{2 h_{K}(\bar{z}-\bar{w})^{2 \bar{h}_{K}}}} .
$$

The product of quasiprimary operators in each replica forms a quasiprimary operator of $C F T^{n}$,

$$
\Phi_{K}(z, \bar{z})=\prod_{j=0}^{n-1} \phi_{k_{j}}(z, \bar{z})
$$

In this case

$$
K=\left\{k_{j}\right\}, \quad \alpha_{K}=\prod_{j=0}^{n-1} \alpha_{k_{j}}, \quad h_{K}=\sum_{j=0}^{n-1} h_{k_{j}}, \quad \bar{h}_{K}=\sum_{j=0}^{n-1} \bar{h}_{k_{j}} .
$$

We should bear in mind that not all of the quasiprimary operators can be written in this form $[1,3]$.

When the intervals are short, at each interval we have the OPE of the twist operators in $C F T^{n}$

$$
\sigma(z, \bar{z}) \tilde{\sigma}(0,0)=c_{n} \sum_{K} d_{K} \sum_{m, r \geq 0} \frac{a_{K}^{m}}{m !} \frac{\bar{a}_{K}^{r}}{r !} \frac{1}{z^{2 h-h_{K}-m} \bar{z}^{2 \bar{h}-\bar{h}_{K}-r}} \partial^{m} \bar{\partial}^{r} \Phi_{K}(0,0),
$$

with the summation $K$ being over all the independent quasiprimary operators of $C F T^{n}$. Here

$$
a_{K}^{m} \equiv \frac{C_{h_{K}+m-1}^{m}}{C_{2 h_{K}+m-1}^{m}}, \quad \bar{a}_{K}^{r} \equiv \frac{C_{\bar{h}_{K}+r-1}^{r}}{C_{2 \bar{h}_{K}+r-1}^{r}},
$$

with the binomial coefficient being $C_{x}^{y}=\frac{\Gamma(x+1)}{\Gamma(y+1) \Gamma(x-y+1)}$. To calculate the $d_{K}$ 's, we may just consider the one interval case $N=1$, and find that $[2,3]$

$$
d_{K}=\frac{1}{\alpha_{K} \ell^{h_{K}+\bar{h}_{K}}} \lim _{z \rightarrow \infty} z^{2 h_{K}} \bar{z}^{2 \bar{h}_{K}}\left\langle\Phi_{K}(z, \bar{z})\right\rangle_{\mathcal{R}_{n, 1}}
$$

with $\alpha_{K}$ being a normalization coefficient in (3.6) and $\ell$ being the length of the interval in (3.4).

To use the OPE of the twist operators, we have to find the quasiprimary operators level by level. As the holomorphic and anti-holomorphic sector are decoupled and similar, we may just focus on the ones in the holomorphic sector. These operators are listed in appendix A. The OPE coefficients $d_{K}$ for these operators are calculated in appendix B.

We would like to consider the case of two short disjoint intervals on the complex plane. We choose $A=[0, \ell] \cup[1,1+\ell]$ with $\ell$ being small, and thus the cross ratio $x=\ell^{2}$ is small too. As shown in [3], the partition function of $C F T^{n}$ is

$$
\begin{aligned}
\operatorname{Tr} \rho_{A}^{n} & =c_{n}^{2} \ell^{-\frac{c}{3}\left(n-\frac{1}{n}\right)}\left(\sum_{K} \alpha_{K} d_{K}^{2} \ell^{2 h_{K}} \sum_{m, p \geq 0}(-)^{m} \frac{(m+p) !}{m ! p !} a_{K}^{m} a_{K}^{p} C_{2 h_{K}+m+p-1}^{m+p} \ell^{m+p}\right)^{2} \\
& =c_{n}^{2} x^{-\frac{c}{6}\left(n-\frac{1}{n}\right)}\left(\sum_{K} \alpha_{K} d_{K}^{2} x^{h_{K}} F\left(h_{K}, h_{K} ; 2 h_{K} ; x\right)\right)^{2}
\end{aligned}
$$


with $K$ being the summation over all holomorphic quasiprimary operators and $F\left(h_{K}, h_{K} ; 2 h_{K} ; x\right)$ being the hypergeometric function. Note that we have used the formula

$$
\sum_{m, p \geq 0}(-)^{m} \frac{(m+p) !}{m ! p !} a_{K}^{m} a_{K}^{p} C_{2 h_{K}+m+p-1}^{m+p} \ell^{m+p}=F\left(h_{K}, h_{K} ; 2 h_{K} ; \ell^{2}\right) .
$$

\subsection{Ordinary CFT}

Firstly we only consider the quasiprimary operators constructed solely by the operators in the Virasoro Verma module. The process in straightforward but tedious,. Some intermediate steps in the calculation can be found in appendix C.

We obtain the Rényi mutual information

$$
I_{n}^{(2)}=I_{n}^{(2) \text { tree }}+I_{n}^{(2) 1-\text { loop }}+I_{n}^{(2) 2-\text { loop }}+I_{n}^{(2) 3-\text { loop }}+\cdots
$$

Here we use the upper symbol (2) to remind that this is the contribution from the Virasoro Verma module. The tree part, or the so-called classical part, being proportional to the central charge $c$, is

$$
\begin{aligned}
I_{n}^{(2) \text { tree }}= & \frac{c(n-1)(n+1)^{2} x^{2}}{144 n^{3}}+\frac{c(n-1)(n+1)^{2} x^{3}}{144 n^{3}}+\frac{c(n-1)(n+1)^{2}\left(1309 n^{4}-2 n^{2}-11\right) x^{4}}{207360 n^{7}} \\
& +\frac{c(n-1)(n+1)^{2}\left(589 n^{4}-2 n^{2}-11\right) x^{5}}{103680 n^{7}} \\
& +\frac{c(n-1)(n+1)^{2}\left(805139 n^{8}-4244 n^{6}-23397 n^{4}-86 n^{2}+188\right) x^{6}}{156764160 n^{11}} \\
& +\frac{c(n-1)(n+1)^{2}\left(244439 n^{8}-1724 n^{6}-9537 n^{4}-86 n^{2}+188\right) x^{7}}{52254720 n^{11}} \\
& +\frac{c(n-1)(n+1)^{2} x^{8}}{1504935936000 n^{15}}\left(6459666587 n^{12}-56285106 n^{10}-312586347 n^{8}-4722748 n^{6}\right. \\
& \left.+10301973 n^{4}+67854 n^{2}-58213\right)+O\left(x^{9}\right) .
\end{aligned}
$$

This is just (2.4) and matches the result in [1, 27, 28]. The quantum 1-loop part, being proportional to $c^{0}$, is

$$
\begin{aligned}
I_{n}^{(2) 1-\text { loop }}= & \frac{(n+1)\left(n^{2}+11\right)\left(3 n^{4}+10 n^{2}+227\right) x^{4}}{3628800 n^{7}} \\
& +\frac{(n+1)\left(109 n^{8}+1495 n^{6}+11307 n^{4}+81905 n^{2}-8416\right) x^{5}}{59875200 n^{9}} \\
& +\frac{(n+1) x^{6}}{523069747200 n^{11}}\left(1444050 n^{10}+19112974 n^{8}+140565305 n^{6}+1000527837 n^{4}\right. \\
& \left.-167731255 n^{2}-14142911\right) \\
& +\frac{(n+1) x^{7}}{1569209241600 n^{13}}\left(5631890 n^{12}+72352658 n^{10}+520073477 n^{8}+3649714849 n^{6}\right. \\
& \left.-767668979 n^{4}-140870807 n^{2}+13778112\right) \\
& +\frac{(n+1) x^{8}}{3766102179840000 n^{15}}\left(16193555193 n^{14}+202784829113 n^{12}+1429840752361 n^{10}\right. \\
& +9916221391201 n^{8}-2370325526301 n^{6}-689741905741 n^{4} \\
& \left.+59604098747 n^{2}+161961045427\right)+O\left(x^{9}\right)
\end{aligned}
$$


which matches the result in [30] to order $x^{8}$. There are also the quantum 2-loop contributions, being proportional to $1 / c$,

$$
\begin{aligned}
I_{n}^{(2) 2-\text { loop }}= & \frac{(n+1)\left(n^{2}-4\right)\left(19 n^{8}+875 n^{6}+22317 n^{4}+505625 n^{2}+5691964\right) x^{6}}{70053984000 n^{11} c} \\
& +\frac{(n+1)\left(n^{2}-4\right) x^{7}}{326918592000 n^{13} c}\left(276 n^{10}+12571 n^{8}+317643 n^{6}+7151253 n^{4}\right. \\
& \left.+79361381 n^{2}-9428724\right) \\
& +\frac{(n+1)\left(n^{2}-4\right) x^{8}}{8002967132160000 n^{15} c}\left(13294413 n^{12}+599852505 n^{10}+15034412216 n^{8}\right. \\
& \left.+336566965090 n^{6}+3691373772429 n^{4}-744486199595 n^{2}-466025361058\right)+O\left(x^{9}\right),
\end{aligned}
$$

and the quantum 3-loop contributions, being proportional to $1 / c^{2}$,

$$
\begin{aligned}
I_{n}^{(2) 3-\text { loop }}= & \frac{(n+1)\left(n^{2}-4\right)\left(n^{2}-9\right) x^{8}}{111152321280000 n^{15} c^{2}}\left(21 n^{10}+1994 n^{8}+105648 n^{6}+4785522 n^{4}\right. \\
& \left.+141534331 n^{2}+2127620484\right)+O(x)^{9} .
\end{aligned}
$$

\subsection{CFT with $W(2,3)$ symmetry}

Taking the contributions only from the $W_{3}$ field into account, we get the mutual information

$$
I_{n}^{(2,3)}=I_{n}^{(2,3) \text { tree }}+I_{n}^{(2,3) 1-\text { loop }}+I_{n}^{(2,3) 2-\text { loop }}+I_{n}^{(2,3) 3-\text { loop }}+\cdots,
$$

where $I_{n}^{(2,3) \text { tree }}$ being the same as (3.15),

$$
\begin{aligned}
I_{n}^{(2,3) 1-\text { loop }}= & \cdots+\frac{(n+1) x^{6}}{1307674368000 n^{11}}\left(3610816 n^{10}+47796776 n^{8}+351567243 n^{6}\right. \\
& \left.+2502467423 n^{4}-412426559 n^{2}+10856301\right) \\
& +\frac{(n+1) x^{7}}{3923023104000 n^{13}}\left(14086574 n^{12}+181019636 n^{10}+1301627579 n^{8}\right. \\
& \left.+9134819803 n^{6}-1856911805 n^{4}+60815461 n^{2}+4299552\right) \\
& +\frac{(n+1) x^{8}}{64023737057280000 n^{15}}\left(275521519443 n^{14}+3451880530003 n^{12}\right. \\
& +24353751911391 n^{10}+168908577430911 n^{8}-38355668351111 n^{6} \\
& \left.+1040661434169 n^{4}-560442831723 n^{2}+2551371092917\right)+O\left(x^{9}\right), \quad
\end{aligned}
$$

with the $\cdots$ being the $x^{4}, x^{5}$ parts of $I_{n}^{(2) 1-\text { loop }}$,

$$
\begin{aligned}
I_{n}^{(2,3) 2-\text { loop }}= & \cdots+\frac{(n+1)\left(n^{2}-4\right) x^{8}}{4001483566080000 c n^{15}}\left(6650397 n^{12}+300093675 n^{10}+7521887359 n^{8}\right. \\
& \left.+168383511650 n^{6}+1847152960191 n^{4}-354725220325 n^{2}-75741834947\right) \\
& +O\left(x^{9}\right),
\end{aligned}
$$

with the $\cdots$ being the $x^{6}, x^{7}$ parts of $I_{n}^{(2) 2-\text { loop }}$, and $I_{n}^{(2,3) 3-\text { loop }}$ being the same as $I_{n}^{(2) 3-\text { loop }}$.

Regarding the above results, several remarks are in order 
- There is no further contributions from the $W$-generated operators to the tree-level result. This is consistent with the fact that there is no higher spin contribution to the classical action in the bulk.

- The 1-loop result $I_{n}^{(2,3) 1-\text { loop }}(3.20)$ is just the summation of $I_{n}^{(2) 1-l o o p}(3.16)$ and $I_{n}^{\text {spin-3,1-loop }}(2.8)$, and is in exact match with holographic computation.

- The 2-loop and 3-loop results are vanishing for $n=2$. This is in accord with the fact that the genus-1 partition function is 1-loop exact. Correspondingly the quantum correction to the gravitational configuration with the asymptotic boundary being genus-1 is 1-loop exact [32].

- The 2-loop corrections are generically nonvanishing for $n \geq 3$. This fact is in accord with the fact that in gravity there exist higher loop corrections to the gravitational configurations with the asymptotic boundary being higher genus [37].

- The 3-loop correction seems to be vanishing at $n=3$. It is not clear if there is a good reason for this fact.

\subsection{CFT with $W(2,4)$ symmetry}

Taking the contributions only from the $W_{4}$ field into account, we get the mutual information

$$
I_{n}^{(2,4)}=I_{n}^{(2,4) \text { tree }}+I_{n}^{(2,4) 1-\text { loop }}+I_{n}^{(2,4) 2-\text { loop }}+I_{n}^{(2,4) 3-\text { loop }}+\cdots,
$$

where $I_{n}^{(2,4) \text { tree }}, I_{n}^{(2,4) 2-\text { loop }}$, and $I_{n}^{(2,4) 3-\text { loop }}$ being the same with $I_{n}^{(2) \text { tree }}(3.15)$, $I_{n}^{(2) 2-\text { loop }}(3.17)$ and $I_{n}^{(2) 3-\text { loop }}(3.18)$ respectively, but

$$
\begin{aligned}
I_{n}^{(2,4) 1-\mathrm{loop}}= & \cdots+\frac{(n+1) x^{8}}{64023737057280000 n^{15}}\left(275290459983 n^{14}+3447342687823 n^{12}\right. \\
& +24307301089071 n^{10}+168575843022951 n^{8}-40294949578091 n^{6} \quad(3.23) \\
& \left.-11722057081371 n^{4}+1032493037037 n^{2}+2876199658597\right)+O\left(x^{9}\right) .
\end{aligned}
$$

with $\cdots$ being the $x^{4}, x^{5}, x^{6}$ and $x^{7}$ parts of $I_{n}^{(2) 1-l o o p}(3.16)$. The 1-loop result is just the summation of $I_{n}^{(2) 1-\text { loop }}(3.16)$ and $I_{n}^{\text {spin-4,1-loop }}(2.9)$, and so is consistent with the bulk result.

\subsection{CFT with $W(2,3,4)$ symmetry}

Taking into account of both the contributions from $W_{3}$ and $W_{4}$ fields, we get the mutual information

$$
I_{n}^{(2,3,4)}=I_{n}^{(2,3,4) \text { tree }}+I_{n}^{(2,3,4) 1-\text { loop }}+I_{n}^{(2,3,4) 2-\text { loop }}+I_{n}^{(2,3,4) 3-\text { loop }}+\cdots,
$$

where $I_{n}^{(2,3,4) \text { tree }}, I_{n}^{(2,3,4) 2-\text { loop }}$, and $I_{n}^{(2,3,4) 3-\text { loop }}$ being the same with $I_{n}^{(2) \text { tree }}(3.15)$, $I_{n}^{(2,3) 2-\text { loop }}(3.21)$, and $I_{n}^{(2) 3-\text { loop }}(3.18)$ being just the summation of $I_{n}^{(2) 1-\text { loop }}$ (3.16), $I_{n, \text { spin-3 }}^{1-\text { loop }}(2.8)$ and $I_{n, \text { spin-4 }}^{1-\text { loop }}(2.9)$. This is consistent with the bulk result as well.

Note that to order $O\left(x^{8}\right)$ we have the relation

$$
I_{n}^{(2,3,4)}=I_{n}^{(2,3)}+I_{n}^{(2,4)}-I_{n}^{(2)},
$$

but it is generally not true for higher order of $x$. 


\section{Conclusion and discussion}

In this paper we investigated the Rényi entropy of two intervals on complex plane with small cross ratio $x$ for $2 \mathrm{D}$ CFT with $W$ symmetry. The results could be written as the expansion of small $x$ to order $x^{8}$, and so only the $W_{3}$ and $W_{4}$ fields, and correspondingly the spin-3 and spin-4 fields in the gravity side, may contribute. We firstly did the calculation in the gravity side using the method in $[27,28,30]$ and got the classical and 1-loop parts of the holographic Rényi entropy. In the calculation we assumed that the classical configurations are not changed by the presence of higher spin fields. Then we calculated in the CFT side using the OPE of twist operators, and found exact match with the gravity results. Our investigation provides a nontrivial test of $\mathrm{HS} / \mathrm{CFT}$ correspondence in $\mathrm{AdS}_{3}$ beyond the genus-1 partition function. Together with the results in [3,30], our study strongly support the holographic computation of Rényi entropy at classical and 1-loop level in both pure $\mathrm{AdS}_{3}$ gravity and the higher spin $\mathrm{AdS}_{3}$ gravities.

Our study shows that there are nonvanishing 2-loop and 3-loop corrections to the Rényi entropies $S_{n}, n>3$. This is related to the fact that the partition function on a higher genus Riemann surface is not 1-loop exact in dual gravity. It is certainly interesting to check the correspondence beyond the 1-loop level. In particular, there is weak sign that the $m$-loop correction with $m \geq 3$ is vanishing for $S_{n}, n=2,3, \cdots, m$. It would be nice to check if this is true in CFT and gravity.

In this work we have considered a general CFT with $W$ symmetry. For a concrete CFT, for example the minimal model that is dual to higher spin gravitational theory with scalars in $\mathrm{AdS}_{3}$ [39-41], we have to consider the contributions from the scalars in the theory. In this so-called Gaberdiel-Gopakumar duality, there are many light states in the CFT which could contribute significantly to the Rényi entropies. However, it is not clear what kind of objects in the bulk they correspond to and if such objects contribute to the bulk computation. We leave this interesting issue to future work.

Recently there has been calculation of Rényi entropy for logarithmic CFT in [42]. The computations in this work and $[3,42]$ are straightforward but quite tedious, and also they are only limited to short interval expansion. It would be nice to find better ways to simplify the computations in order to extend the discussions to higher levels. General calculations beyond short interval expansion would also be desirable.

Another interesting question is about the relation between different Rényi entropies $S_{n}$. As it is well-known that for two intervals on complex plane case $S_{2}$ is the genus-1 partition function which could be computed in CFT in other ways [1], and $S_{n}$ is expected to be the genus- $(n-1)$ partition function of the CFT. From the works [3, 42], once the genus-1 partition function is in match with the 1-loop bulk partition function, so do the higher Rényi entropies $S_{n}(n>2)$ match with the bulk partition function, at least to 1-loop. It would be great to see if or not this is true in general.

In this work, we focused on the entanglement entropy in the ground state of CFT, without turning on the chemical potential for $W$ charges. In [43, 44], the higher spin entanglement entropy has been discussed from gravity side. It would be interesting to investigate the higher spin Rényi entropies holographically or study them in CFT. 


\section{Acknowledgments}

We thank Matthew Headrick for his Mathematica code Virasoro.nb that could be downloaded at his personal homepage http://people.brandeis.edu/ headrick/Mathematica/in dex.html. BC would like to thank NCTS, Taiwan for hospitality during the final stage of this work. We thank the anonymous referee of the paper for the valuable comments and suggestions. The work was in part supported by NSFC Grant No. 11275010, No. 11335012 and No. 11325522. JJZ was also in part supported by the Scholarship Award for Excellent Doctoral Student granted by the Ministry of Education of China.

\section{A Counting of quasiprimary operators}

In this appendix we count the quasiprimary operators that are needed in the calculation, and get their normalization constant $\alpha_{K}$ defined in (3.6). At the first step, we work out the holomorphic quasiprimary operators constructed by the operators in the Virasoro Verma module to level eight for an ordinary CFT and then for the $C F T^{n}$. Next we find the additional holomorphic quasiprimary operators in the presence of $W$ fields. For the original CFT before taking the replica, the partition function of the unit operator 1 is

$$
\operatorname{tr} x^{L_{0}}=\prod_{m=2}^{\infty} \frac{1}{1-x^{m}}=1+x^{2}+x^{3}+2 x^{4}+2 x^{5}+4 x^{6}+4 x^{7}+7 x^{8}+O\left(x^{9}\right),
$$

where tr counts the number of holomorphic operators. So the number of linearly independent holomorphic quasiprimary operators \# at each level $L_{0}$ is

\begin{tabular}{|l|l|l|l|l|l|l|l|l|l|}
\hline$L_{0}$ & 0 & 2 & 3 & 4 & 5 & 6 & 7 & 8 & $\cdots$ \\
\hline$\#$ & 1 & 1 & 0 & 1 & 0 & 2 & 0 & 3 & $\cdots$ \\
\hline
\end{tabular}

With some efforts, we could get the quasiprimary operators listed as follows.

- At level 0 , it is of course the identity 1 with $\alpha_{1}=1$.

- At level 2, it is $T$ with $\alpha_{T}=\frac{c}{2}$.

- At level 4 , it is $\mathcal{A}=(T T)-\frac{3}{10} \partial^{2} T$ with $\alpha_{\mathcal{A}}=\frac{c(5 c+22)}{10}$.

- At level 6, they are

$$
\begin{aligned}
\mathcal{B} & =(\partial T \partial T)-\frac{4}{5}\left(T \partial^{2} T\right)+\frac{23}{210} \partial^{4} T \\
\mathcal{D} & =\mathcal{C}+\frac{93}{70 c+29} \mathcal{B}
\end{aligned}
$$

with

$$
\mathcal{C}=(T(T T))-\frac{9}{10}\left(T \partial^{2} T\right)+\frac{4}{35} \partial^{4} T .
$$

Their normalization constants are respectively

$$
\alpha_{\mathcal{B}}=\frac{36 c(70 c+29)}{175}
$$




$$
\alpha_{\mathcal{D}}=\frac{3 c(2 c-1)(5 c+22)(7 c+68)}{4(70 c+29)} .
$$

- At level 8, they are

$$
\begin{aligned}
& \mathcal{E}=\left(\partial^{2} T \partial^{2} T\right)-\frac{10}{9}\left(\partial T \partial^{3} T\right)+\frac{10}{63}\left(T \partial^{4} T\right)-\frac{13}{2268} \partial^{6} T \\
& \mathcal{H}=\mathcal{F}+\frac{9(140 c+83)}{50(105 c+11)} \mathcal{E} \\
& \mathcal{I}=\mathcal{G}+\frac{81(35 c-51)}{100(105 c+11)} \mathcal{E}+\frac{12(465 c-127)}{5 c(210 c+661)-251} \mathcal{H},
\end{aligned}
$$

with

$$
\begin{aligned}
& \mathcal{F}=(T(\partial T \partial T))-\frac{4}{5}\left(T\left(T \partial^{2} T\right)\right)-\frac{1}{5}\left(\partial T \partial^{3} T\right)+\frac{47}{210}\left(T \partial^{4} T\right)-\frac{29}{2520} \partial^{6} T \\
& \mathcal{G}=(T(T(T T)))-\frac{9}{5}\left(T\left(T \partial^{2} T\right)\right)+\frac{3}{10}\left(\partial T \partial^{3} T\right)+\frac{29}{70}\left(T \partial^{4} T\right)-\frac{41}{1680} \partial^{6} T .
\end{aligned}
$$

The corresponding normalization constants are respectively

$$
\begin{aligned}
\alpha_{\mathcal{E}} & =\frac{22880 c(105 c+11)}{1323}, \\
\alpha_{\mathcal{H}} & =\frac{26 c(5 c+22)(5 c(210 c+661)-251)}{125(105 c+11)}, \\
\alpha_{\mathcal{I}} & =\frac{3 c(2 c-1)(3 c+46)(5 c+3)(5 c+22)(7 c+68)}{2(5 c(210 c+661)-251)} .
\end{aligned}
$$

For the $C F T^{n}$, we consider the partition function

$$
\begin{aligned}
\operatorname{tr} x^{L_{0}}=\prod_{m=2}^{\infty} \frac{1}{\left(1-x^{m}\right)^{n}}= & +n x^{2}+n x^{3}+\frac{n(n+3)}{2} x^{4}+n(n+1) x^{5}+\frac{n(n+1)(n+11)}{6} x^{6} \\
& +\frac{n\left(n^{2}+5 n+2\right)}{2} x^{7}+\frac{n(n+3)\left(n^{2}+27 n+14\right)}{24} x^{8}+O\left(x^{9}\right),(\text { A. }
\end{aligned}
$$

where tr counts the holomorphic operators of the $C F T^{n}$. The number of linear independent holomorphic quasiprimary operators \# at each level $L_{0}$ is

\begin{tabular}{|c|c|c|c|c|c|c|c|c|c|c|}
\hline$L_{0}$ & 0 & 1 & 2 & 3 & 4 & 5 & 6 & 7 & 8 & $\cdots$ \\
\hline$\#$ & 1 & 0 & $n$ & 0 & $\frac{n(n+1)}{2}$ & $\frac{n(n-1)}{2}$ & $\frac{n(n+1)(n+5)}{6}$ & $\frac{n(n-1)(2 n+5)}{6}$ & $\frac{n(n+1)\left(n^{2}+17 n+18\right)}{24}$ & $\cdots$ \\
\hline
\end{tabular}

The quasiprimary operators are listed in the following table, in which $j_{i}$ 's are integer, taking values between 0 and $n-1$. 


\begin{tabular}{|c|c|c|c|}
\hline$L_{0}$ & quasiprimary operators & degeneracies & $\#$ \\
\hline 0 & 1 & 1 & 1 \\
\hline 2 & $T_{j}$ & $n$ & $n$ \\
\hline \multirow{2}{*}{4} & $\mathcal{A}_{j}$ & $n$ & \multirow{2}{*}{$\frac{n(n+1)}{2}$} \\
\hline & $T_{j_{1}} T_{j_{2}}$ with $j_{1}<j_{2}$ & $\frac{n(n-1)}{2}$ & \\
\hline 5 & $\mathcal{J}_{j_{1} j_{2}}$ with $j_{1}<j_{2}$ & $\frac{n(n-1)}{2}$ & $\frac{n(n-1)}{2}$ \\
\hline \multirow{5}{*}{6} & $\mathcal{B}_{j}$ & $n$ & \multirow{5}{*}{$\frac{n(n+1)(n+5)}{6}$} \\
\hline & $\mathcal{D}_{j}$ & $n$ & \\
\hline & $T_{j_{1}} \mathcal{A}_{j_{2}}$ with $j_{1} \neq j_{2}$ & $n(n-1)$ & \\
\hline & $\mathcal{K}_{j_{1} j_{2}}$ with $j_{1}<j_{2}$ & $\frac{n(n-1)}{2}$ & \\
\hline & $T_{j_{1}} T_{j_{2}} T_{j_{3}}$ with $j_{1}<j_{2}<j_{3}$ & $\frac{n(n-1)(n-2)}{6}$ & \\
\hline \multirow{4}{*}{7} & $\mathcal{L}_{j_{1} j_{2}}$ with $j_{1} \neq j_{2}$ & $n(n-1)$ & \multirow{4}{*}{$\frac{n(n-1)(2 n+5)}{6}$} \\
\hline & $\mathcal{M}_{j_{1} j_{2}}$ with $j_{1}<j_{2}$ & $\frac{n(n-1)}{2}$ & \\
\hline & $T_{j_{1}} \mathcal{J}_{j_{2} j_{3}}$ with $j_{1}<j_{2}<j_{3}$ & \multirow{2}{*}{$\frac{n(n-1)(n-2)}{3}$} & \\
\hline & $\mathcal{N}_{j_{1} j_{2} j_{3}}$ with $j_{1}<j_{2}<j_{3}$ & & \\
\hline \multirow{13}{*}{8} & $\mathcal{E}_{j}$ & $n$ & \multirow{13}{*}{$\frac{n(n+1)\left(n^{2}+17 n+18\right)}{24}$} \\
\hline & $\mathcal{H}_{j}$ & $n$ & \\
\hline & $\mathcal{I}_{j}$ & $n$ & \\
\hline & $T_{j_{1}} \mathcal{B}_{j_{2}}$ with $j_{1} \neq j_{2}$ & $n(n-1)$ & \\
\hline & $T_{j_{1}} \mathcal{D}_{j_{2}}$ with $j_{1} \neq j_{2}$ & $n(n-1)$ & \\
\hline & $\mathcal{A}_{j_{1}} \mathcal{A}_{j_{2}}$ with $j_{1}<j_{2}$ & $\frac{n(n-1)}{2}$ & \\
\hline & $\mathcal{O}_{j_{1} j_{2}}$ with $j_{1} \neq j_{2}$ & $n(n-1)$ & \\
\hline & $\mathcal{P}_{j_{1} j_{2}}$ with $j_{1}<j_{2}$ & $\frac{n(n-1)}{2}$ & \\
\hline & $T_{j_{1}} T_{j_{2}} \mathcal{A}_{j_{3}}$ with $j_{1}<j_{2}, j_{1} \neq j_{3}$ and $j_{2} \neq j_{3}$ & $\frac{n(n-1)(n-2)}{2}$ & \\
\hline & $T_{j_{1}} \mathcal{K}_{j_{2} j_{3}}$ with $j_{1}<j_{2}<j_{3}$ & \multirow{3}{*}{$\frac{n(n-1)(n-2)}{2}$} & \\
\hline & $\mathcal{Q}_{j_{1} j_{2} j_{3}}$ with $j_{1}<j_{2}<j_{3}$ & & \\
\hline & $\mathcal{R}_{j_{1} j_{2} j_{3}}$ with $j_{1}<j_{2}<j_{3}$ & & \\
\hline & $T_{j_{1}} T_{j_{2}} T_{j_{3}} T_{j_{4}}$ with $j_{1}<j_{2}<j_{3}<j_{4}$ & $\frac{n(n-1)(n-2)(n-3)}{24}$ & \\
\hline$\cdots$ & $\cdots$ & $\cdots$ & $\cdots$ \\
\hline
\end{tabular}

In the above table, there are several new quasiprimary operators, which could not be written as the product of the quasiprimary operators in different replicas. At level five and six, they are

$$
\begin{aligned}
& \mathcal{J}_{j_{1} j_{2}}=T_{j_{1}} i \partial T_{j_{2}}-i \partial T_{j_{1}} T_{j_{2}} \\
& \mathcal{K}_{j_{1} j_{2}}=\partial T_{j_{1}} \partial T_{j_{2}}-\frac{2}{5}\left(T_{j_{1}} \partial^{2} T_{j_{2}}+\partial^{2} T_{j_{1}} T_{j_{2}}\right) .
\end{aligned}
$$


At level seven they are

$$
\begin{aligned}
\mathcal{L}_{j_{1} j_{2}} & =T_{j_{1}} i \partial \mathcal{A}_{j_{2}}-2 i \partial T_{j_{1}} \mathcal{A}_{j_{2}}, \\
\mathcal{M}_{j_{1} j_{2}} & =i \partial T_{j_{1}} \partial^{2} T_{j_{2}}-\partial^{2} T_{j_{1}} i \partial T_{j_{2}}+\frac{2}{9}\left(i \partial^{3} T_{j_{1}} T_{j_{2}}-T_{j_{1}} i \partial^{3} T_{j_{2}}\right), \\
\mathcal{N}_{j_{1} j_{2} j_{3}} & =T_{j_{1}}\left(T_{j_{2}} i \partial T_{j_{3}}+i \partial T_{j_{2}} T_{j_{3}}\right)-2 i \partial T_{j_{1}} T_{j_{2}} T_{j_{3}} .
\end{aligned}
$$

At level eight they are

$$
\begin{aligned}
\mathcal{O}_{j_{1} j_{2}} & =\partial T_{j_{1}} \partial \mathcal{A}_{j_{2}}-\frac{2}{9} T_{j_{1}} \partial^{2} \mathcal{A}_{j_{2}}-\frac{4}{5} \partial^{2} T_{j_{1}} \mathcal{A}_{j_{2}} \\
\mathcal{P}_{j_{1} j_{2}} & =\partial^{2} T_{j_{1}} \partial^{2} T_{j_{2}}-\frac{5}{9}\left(\partial T_{j_{1}} \partial^{3} T_{j_{2}}+\partial^{3} T_{j_{1}} \partial T_{j_{2}}\right)+\frac{5}{63}\left(T_{j_{1}} \partial^{4} T_{j_{2}}+\partial^{4} T_{j_{1}} T_{j_{2}}\right) \\
\mathcal{Q}_{j_{1} j_{2} j_{3}} & =T_{j_{2}} K_{j_{3} j_{1}}-\frac{2}{9} T_{j_{1}} K_{j_{2} j_{3}}, \\
\mathcal{R}_{j_{1} j_{2} j_{3}} & =T_{j_{3}} K_{j_{1} j_{2}}-\frac{2}{11}\left(T_{j_{1}} K_{j_{2} j_{3}}+T_{j_{2}} K_{j_{3} j_{1}}\right)
\end{aligned}
$$

The normalization factors of these orthogonalized quasiprimary operators are respectively

$$
\begin{aligned}
& \alpha_{T T}=\frac{c^{2}}{4}, \quad \alpha_{\mathcal{J}}=2 c^{2}, \quad \alpha_{T \mathcal{A}}=\frac{c^{2}(5 c+22)}{20}, \quad \alpha_{\mathcal{K}}=\frac{36 c^{2}}{5}, \\
& \alpha_{T T T}=\frac{c^{3}}{8}, \quad \alpha_{\mathcal{L}}=\frac{6 c^{2}(5 c+22)}{5}, \quad \alpha_{\mathcal{M}}=\frac{880 c^{2}}{9} \\
& \alpha_{T \mathcal{J}}=c^{3}, \quad \alpha_{\mathcal{N}}=3 c^{3}, \quad \alpha_{T \mathcal{B}}=\frac{18 c^{2}(70 c+29)}{175}, \\
& a_{T \mathcal{D}}=\frac{3 c^{2}(2 c-1)(5 c+22)(7 c+68)}{8(70 c+29)} \\
& \alpha_{\mathcal{A A}}=\frac{c^{2}(5 c+22)^{2}}{100}, \quad \alpha_{\mathcal{O}}=\frac{728 c^{2}(5 c+22)}{225}, \\
& \alpha_{\mathcal{P}}=\frac{57200 c^{2}}{63}, \quad \alpha_{T T \mathcal{A}}=\frac{c^{3}(5 c+22)}{40}, \quad \alpha_{T \mathcal{K}}=\frac{18 c^{3}}{5}, \\
& \alpha_{\mathcal{Q}}=\frac{154 c^{3}}{45}, \quad \alpha_{\mathcal{R}}=\frac{182 c^{3}}{55}, \quad \alpha_{T T T T}=\frac{c^{4}}{16} .
\end{aligned}
$$

For a CFT with $W$ symmetry, we should consider additional contributions, and the partition function is

$$
\operatorname{tr} x^{L_{0}}=\prod_{s} \prod_{m=s}^{\infty} \frac{1}{1-x^{m}}
$$

where the tr counts the number of the holomorphic operators again, and $s$ denotes all the possible spins in the theory. If the theory has $W(2,3)$ symmetry, it is

$$
\operatorname{tr} x^{L_{0}}=\frac{1}{1-x^{2}} \prod_{m=3}^{\infty} \frac{1}{\left(1-x^{m}\right)^{2}}=1+x^{2}+2 x^{3}+3 x^{4}+4 x^{5}+8 x^{6}+10 x^{7}+17 x^{8}+O\left(x^{9}\right),
$$

and for $C F T^{n}$ it would be

$$
\operatorname{tr} x^{L_{0}}=\frac{1}{\left(1-x^{2}\right)^{n}} \prod_{m=3}^{\infty} \frac{1}{\left(1-x^{m}\right)^{2 n}}
$$




$$
\begin{aligned}
= & 1+n x^{2}+2 n x^{3}+\frac{n(n+5)}{2} x^{4}+2 n(n+1) x^{5}+\frac{n\left(n^{2}+27 n+20\right)}{6} x^{6} \\
& +n\left(n^{2}+7 n+2\right) x^{7}+\frac{n(n+3)\left(n^{2}+75 n+26\right)}{24} x^{8}+O\left(x^{9}\right) .
\end{aligned}
$$

The numbers of independent quasiprimary operators for the CFT and the $C F T^{n}$ are listed as follows.

\begin{tabular}{|c|c|c|c|c|c|c|c|c|c|c|}
\hline$L_{0}$ & 0 & 1 & 2 & 3 & 4 & 5 & 6 & 7 & 8 & $\cdots$ \\
\hline CFT & 1 & 0 & 1 & 1 & 1 & 1 & 4 & 2 & 7 & $\cdots$ \\
\hline$C F T^{n}$ & 1 & 0 & $n$ & $n$ & $\frac{n(n+1)}{2}$ & $\frac{n(3 n-1)}{2}$ & $\frac{n\left(n^{2}+15 n+8\right)}{6}$ & $\frac{n\left(5 n^{2}+15 n-8\right)}{6}$ & $\frac{n(n+1)\left(n^{2}+53 n+30\right)}{24}$ & $\cdots$ \\
\hline
\end{tabular}

If the theory has $W(2,4)$ symmetry, it is

$\operatorname{tr} x^{L_{0}}=\frac{1}{\left(1-x^{2}\right)\left(1-x^{3}\right)} \prod_{m=4}^{\infty} \frac{1}{\left(1-x^{m}\right)^{2}}=1+x^{2}+x^{3}+3 x^{4}+3 x^{5}+6 x^{6}+7 x^{7}+13 x^{8}+O\left(x^{9}\right)$,

and for $C F T^{n}$ it would be

$$
\begin{aligned}
\operatorname{tr} x^{L_{0}}= & \frac{1}{\left(1-x^{2}\right)^{n}\left(1-x^{3}\right)^{n}} \prod_{m=4}^{\infty} \frac{1}{\left(1-x^{m}\right)^{2 n}} \\
= & 1+n x^{2}+n x^{3}+\frac{n(n+5)}{2} x^{4}+n(n+2) x^{5}+\frac{n(n+1)(n+17)}{6} x^{6} \\
& +\frac{n\left(n^{2}+9 n+4\right)}{2} x^{7}+\frac{n\left(n^{3}+42 n^{2}+191 n+78\right)}{24} x^{8}+O\left(x^{9}\right) .
\end{aligned}
$$

Then we can get the numbers of independent quasiprimary operators for the CFT and the $C F T^{n}$ listed as follows.

\begin{tabular}{|c|c|c|c|c|c|c|c|c|c|c|}
\hline$L_{0}$ & 0 & 1 & 2 & 3 & 4 & 5 & 6 & 7 & 8 & $\cdots$ \\
\hline $\mathrm{CFT}$ & 1 & 0 & 1 & 0 & 2 & 0 & 3 & 1 & 6 & $\cdots$ \\
\hline$C F T^{n}$ & 1 & 0 & $n$ & 0 & $\frac{n(n+3)}{2}$ & $\frac{n(n-1)}{2}$ & $\frac{n\left(n^{2}+12 n+5\right)}{6}$ & $\frac{n(n+5)(2 n-1)}{6}$ & $\frac{n\left(n^{3}+30 n^{2}+83 n+30\right)}{24}$ & $\cdots$ \\
\hline
\end{tabular}

If the theory has $W(2,3,4)$ symmetry, it is

$$
\operatorname{tr} x^{L_{0}}=\frac{1}{\left(1-x^{2}\right)\left(1-x^{3}\right)^{2}} \prod_{m=4}^{\infty} \frac{1}{\left(1-x^{m}\right)^{3}}=1+x^{2}+2 x^{3}+4 x^{4}+5 x^{5}+10 x^{6}+14 x^{7}+25 x^{8}+O\left(x^{9}\right),
$$

and for $C F T^{n}$ it would be

$$
\begin{aligned}
\operatorname{tr} x^{L_{0}}= & \frac{1}{\left(1-x^{2}\right)^{n}\left(1-x^{3}\right)^{2 n}} \prod_{m=4}^{\infty} \frac{1}{\left(1-x^{m}\right)^{3 n}} \\
= & 1+n x^{2}+2 n x^{3}+\frac{n(n+7)}{2} x^{4}+n(2 n+3) x^{5}+\frac{n\left(n^{2}+33 n+26\right)}{6} x^{6} \\
& +n\left(n^{2}+10 n+3\right) x^{7}+\frac{n\left(n^{3}+90 n^{2}+395 n+114\right)}{24} x^{8}+O\left(x^{9}\right)
\end{aligned}
$$

Then the numbers of independent quasiprimary operators for the CFT and the $C F T^{n}$ are listed as follows. 


\begin{tabular}{|c|c|c|c|c|c|c|c|c|c|c|}
\hline$L_{0}$ & 0 & 1 & 2 & 3 & 4 & 5 & 6 & 7 & 8 & $\cdots$ \\
\hline $\mathrm{CFT}^{n}$ & 1 & 0 & 1 & 1 & 2 & 1 & 5 & 4 & 11 & $\cdots$ \\
\hline$C F T^{n}$ & 1 & 0 & $n$ & $n$ & $\frac{n(n+3)}{2}$ & $\frac{n(3 n-1)}{2}$ & $\frac{n\left(n^{2}+21 n+8\right)}{6}$ & $\frac{n\left(5 n^{2}+27 n-8\right)}{6}$ & $\frac{n\left(n^{3}+66 n^{2}+155 n+42\right)}{24}$ & $\cdots$ \\
\hline
\end{tabular}

There are large numbers of the quasiprimary operators from $W$ fields. However, most of them have vanishing coefficients $d_{K}$ so that they do not contribute to the OPE of the twist operators.

For the CFT with $W(2,3)\left(W_{3}\right)$ symmetry, we denote the extra holomorphic and antiholomorphic primary operator as $W(z)=W_{3}(z)$ and $\bar{W}(\bar{z})=\bar{W}_{3}(\bar{z})$ which have conformal weight $(3,0)$ and $(0,3)$ respectively. In this case to level eight for $C F T^{n}$, the additional holomorphic quasiprimary operators with nonvanishing coefficients $d_{K}$ are listed as below.

\begin{tabular}{|c|c|c|}
\hline$L_{0}$ & quasiprimary operators & degeneracies \\
\hline 6 & $W_{j_{1}} W_{j_{2}}$ with $j_{1}<j_{2}$ & $\frac{n(n-1)}{2}$ \\
\hline 7 & $\mathcal{U}_{j_{1} j_{2}}$ with $j_{1}<j_{2}$ & $\frac{n(n-1)}{2}$ \\
\hline \multirow{3}{*}{8} & $W_{j_{1}} \mathcal{S}_{j_{2}}$ with $j_{1} \neq j_{2}$ & $n(n-1)$ \\
\cline { 2 - 3 } & $\mathcal{V}_{j_{1} j_{2}}$ with $j_{1}<j_{2}$ & $\frac{n(n-1)}{2}$ \\
\cline { 2 - 3 } & $T_{j_{1}} W_{j_{2}} W_{j_{3}}$ with $j_{1} \neq j_{2}, j_{1} \neq j_{3}$ and $j_{2}<j_{3}$ & $\frac{n(n-1)(n-2)}{2}$ \\
\hline$\cdots$ & $\cdots$ & $\cdots$ \\
\hline
\end{tabular}

Here we have

$$
\begin{aligned}
\mathcal{S} & =(T W)-\frac{3}{14} \partial^{2} W \\
\mathcal{U}_{j_{1} j_{2}} & =W_{j_{1}} i \partial W_{j_{2}}-i \partial W_{j_{1}} W_{j_{2}}, \\
\mathcal{V}_{j_{1} j_{2}} & =\partial W_{j_{1}} \partial W_{j_{2}}-\frac{2}{7}\left(W_{j_{1}} \partial^{2} W_{j_{2}}+\partial^{2} W_{j_{1}} W_{j_{2}}\right) .
\end{aligned}
$$

As we mentioned there are actually some other quasiprimary operators, for examples $W_{j}$ at level three and $T_{j_{1}} W_{j_{2}}$ with $j_{1} \neq j_{2}$ at level five, but all of them have vanishing coefficients $d_{K}$, and so do not contribute to the OPE of twist operators. The normalization factor of $W(z)$ is $\alpha_{W}=\frac{c}{3}$ such that the normalization factors of the above quasiprimary operators are respectively

$$
\begin{aligned}
& \alpha_{\mathcal{S}}=\frac{c(7 c+114)}{42}, \quad \alpha_{W W}=\frac{c^{2}}{9}, \quad \alpha_{\mathcal{U}}=\frac{4 c^{2}}{3}, \\
& \alpha_{W \mathcal{S}}=\frac{c^{2}(7 c+114)}{126}, \quad \quad \alpha_{\mathcal{V}}=\frac{52 c^{2}}{7}, \quad \alpha_{T W W}=\frac{c^{3}}{18} .
\end{aligned}
$$

Obviously, if we only consider the operators up to level 6, the quasiprimary operators from $W$ fields are somehow trivial, without mixing with the stress tensor.

For the CFT with $W(2,4)$ symmetry, we denote the extra holomorphic and antiholomorphic primary operators as $W_{4}(z)$ and $\bar{W}_{4}(\bar{z})$, which has conformal weight $(4,0)$ and $(0,4)$ respectively. In this case to level eight for $C F T^{n}$, the additional holomorphic quasiprimary operators are $W_{4 j_{1}} W_{4 j_{2}}$ with $j_{1}<j_{2}$. We choose the normalization such that

$$
\alpha_{W_{4}}=\alpha_{W_{4} W_{4}}=1 \text {. }
$$


For the CFT with $W(2,3,4)\left(W_{4}\right)$ symmetry, the extra holomorphic quasiprimary operators to level eight consist of the ones from $W_{3}$ field and the ones from $W_{4}$ field listed above. One may wonder if there exist the quasiprimary operators that are the mixtures of the stress tensor, $W_{3}$ and $W_{4}$ fields. Such kind of operators do exist, for example $W_{3 j_{1}} W_{4 j_{2}}$ with $j_{1} \neq j_{2}$ at level seven, but they having vanishing coefficients $d_{K}$ and do not contribute.

\section{B The coefficients $d_{K}$}

The calculation of the OPE coefficients $d_{K}$ for the quasiprimary operators is straightforward but tedious $[2,3]$. For brevity, we only outline the process and then give the results. Firstly we need to determine how the holomorphic operators $T(z), \mathcal{A}(z), \mathcal{B}(z), \mathcal{D}(z), \mathcal{E}(z), \mathcal{H}(z)$ and $\mathcal{I}(z)$ transform under the coordinate transformation $z \rightarrow f(z)$ with $f(z)$ being a general holomorphic function of $z$. We denote the Schwarz derivative as

$$
s(z) \equiv \frac{f^{\prime \prime \prime}(z)}{f^{\prime}(z)}-\frac{3}{2}\left(\frac{f^{\prime \prime}(z)}{f^{\prime}(z)}\right)^{2},
$$

and for simplicity we use the shorthands

$$
\begin{array}{llll}
f=f(z), & f^{\prime}=f^{\prime}(z), & f^{\prime \prime}=f^{\prime \prime}(z), & \ldots \\
s=s(z), & s^{\prime}=s^{\prime}(z), & s^{\prime \prime}=s^{\prime \prime}(z), & \ldots .
\end{array}
$$

The transformations of $T$ and $\mathcal{A}$ are

$$
\begin{aligned}
& T(z)=f^{\prime 2} T(f)+\frac{c}{12} s, \\
& \mathcal{A}(z)=f^{\prime 4} \mathcal{A}(f)+\frac{5 c+22}{30} s\left(f^{\prime 2} T(f)+\frac{c}{24} s\right) .
\end{aligned}
$$

The transformation of one single operator include the homogeneous terms, which are proportional to nonidentity quasiprimary operators or derivatives thereof, and the inhomogeneous terms which are proportional to the identity operator. For examples, the transformation of $T(z)$ includes one homogeneous term $f^{\prime 2} T(f)$ and one inhomogeneous term $\frac{c}{12} s$, while the transformation of $\mathcal{A}(z)$ includes two homogeneous terms $f^{\prime 4} \mathcal{A}(f)+\frac{5 c+22}{30} f^{\prime 2} s T(f)$ and one inhomogeneous term $\frac{c(5 c+22) s^{2}}{720}$. The transformation of $\mathcal{B}(z)$ is complicated

$$
\begin{aligned}
\mathcal{B}(z)= & f^{\prime 6} \mathcal{B}(f)-\frac{8}{5} f^{\prime 4} s \mathcal{A}(f) \\
& -\frac{1}{1050}\left(28(5 c+22) f^{\prime 2} s^{2}+(70 c+29)\left(f^{\prime 2} s^{\prime \prime}-5 f^{\prime} f^{\prime \prime} s^{\prime}+5 f^{\prime \prime 2} s\right)\right) T(f) \\
& +\frac{70 c+29}{420} f^{\prime 2}\left(f^{\prime} s^{\prime}-2 f^{\prime \prime} s\right) \partial T(f)-\frac{70 c+29}{1050} f^{\prime 4} s \partial^{2} T(f) \\
& -\frac{c}{50400}\left(744 s^{3}+(70 c+29)\left(4 s s^{\prime \prime}-5 s^{\prime 2}\right)\right)
\end{aligned}
$$

and the transformation of $\mathcal{D}(z)$ is relatively easy

$$
\mathcal{D}(z)=f^{\prime 6} \mathcal{D}(f)+\frac{(2 c-1)(7 c+68)}{70 c+29} s\left(\frac{5}{4} f^{\prime 4} \mathcal{A}(f)+\frac{5 c+22}{48} s\left(f^{\prime 2} T(f)+\frac{c}{36} s\right)\right) .
$$


In the following discussion we only need the inhomogeneous terms of the transformations of $\mathcal{E}(z), \mathcal{H}(z)$ and $\mathcal{I}(z)$, and explicitly we have

$$
\begin{aligned}
\mathcal{E}(z)= & f^{\prime 8} \mathcal{E}(f)+\cdots \\
& +\frac{c}{952560}\left(23452 s^{4}+2255 s\left(4 s s^{\prime \prime}-5 s^{2}\right)+(105 c+11)\left(10 s s^{(4)}-70 s^{\prime} s^{\prime \prime \prime}+63 s^{\prime \prime 2}\right)\right) \\
\mathcal{H}(z)= & f^{\prime 8} \mathcal{H}(f)+\cdots \\
& -\frac{c(5 c+22)}{1296000(105 c+11)} s\left(104(465 c-127) s^{3}+3(5 c(210 c+661)-251)\left(4 s s^{\prime \prime}-5 s^{2}\right)\right), \\
\mathcal{I}(z)= & f^{\prime 8} \mathcal{I}(f)+\cdots+\frac{c(2 c-1)(3 c+46)(5 c+3)(5 c+22)(7 c+68)}{20736(5 c(210 c+661)-251)} s^{4}
\end{aligned}
$$

with the.. 's represent some homogeneous terms.

The coefficients $d_{K}$ for identity and quasiprmary operators at only one replica could be calculated easily as

$$
\begin{aligned}
& d_{1}=1, \quad d_{T}=\frac{n^{2}-1}{12 n^{2}}, \quad d_{\mathcal{A}}=\frac{\left(n^{2}-1\right)^{2}}{288 n^{4}}, \quad d_{\mathcal{B}}=-\frac{\left(n^{2}-1\right)^{2}\left(2 n^{2}(35 c+61)-93\right)}{10368 n^{6}(70 c+29)} \\
& d_{\mathcal{D}}=\frac{\left(n^{2}-1\right)^{3}}{10368 n^{6}}, \quad d_{\mathcal{E}}=\frac{\left(n^{2}-1\right)^{2}\left(11340 n^{4} c+11561 n^{4}-16236 n^{2}+5863\right)}{65894400 n^{8}(105 c+11)}, \\
& d_{\mathcal{H}}=-\frac{\left(n^{2}-1\right)^{3}\left(3150 n^{2} c^{2}+\left(15960 n^{2}-6045\right) c-\left(2404 n^{2}-1651\right)\right)}{539136 n^{8}(5 c(210 c+661)-251)}, \quad d_{\mathcal{I}}=\frac{\left(n^{2}-1\right)^{4}}{497664 n^{8}} .
\end{aligned}
$$

For quasiprimary operators that can be factorized as two nonidentity quasiprimary operators at two different replicas, we need the orthogonal relation of the operators. And the final results are

$$
\begin{aligned}
d_{T T}^{j_{1} j_{2}}= & \frac{1}{8 n^{4} c} \frac{1}{s_{j_{1} j_{2}}^{4}}+\frac{\left(n^{2}-1\right)^{2}}{144 n^{4}}, \quad d_{T \mathcal{A}}^{j_{1} j_{2}}=\frac{n^{2}-1}{96 n^{6} c} \frac{1}{s_{j_{1} j_{2}}^{4}}+\frac{\left(n^{2}-1\right)^{3}}{3456 n^{6}}, \\
d_{T \mathcal{B}}^{j_{1} j_{2}}= & \frac{5\left(n^{2}-1\right)}{6912 n^{8} c} \frac{1}{s_{j_{1} j_{2}}^{6}}-\frac{\left(n^{2}-1\right)\left(140\left(n^{2}+2\right) c+337 n^{2}-163\right)}{6912 n^{8} c(70 c+29)} \frac{1}{s_{j_{1} j_{2}}^{4}} \\
& -\frac{\left(n^{2}-1\right)^{3}\left(2 n^{2}(35 c+61)-93\right)}{124416 n^{8}(70 c+29)}, \\
d_{T \mathcal{D}}^{j_{1} j_{2}}= & \frac{\left(n^{2}-1\right)^{2}}{2304 n^{8} c} \frac{1}{s_{j_{1} j_{2}}^{4}}+\frac{\left(n^{2}-1\right)^{4}}{124416 n^{8}}, \quad d_{\mathcal{A} \mathcal{A}}^{j_{1} j_{2}}=\frac{5}{128 n^{8} c(5 c+22)} \frac{1}{s_{j_{1} j_{2}}^{8}}+\frac{\left(n^{2}-1\right)^{2}}{1152 n^{8} c} \frac{1}{s_{j_{1} j_{2}}^{4}}+\frac{\left(n^{2}-1\right)^{4}}{82944 n^{8}},
\end{aligned}
$$

where we have defined $s_{j_{1} j_{2}} \equiv \sin \frac{\pi\left(j_{1}-j_{2}\right)}{n}$. For quasiprimary operators that can be factorized as three and four nonidentity quasiprimary operators at different replicas, we need the correlation functions of three and four quasiprimary operators. The correlation functions we need are

$$
\begin{aligned}
\left\langle T\left(f_{1}\right) T\left(f_{2}\right) T\left(f_{3}\right)\right\rangle_{C}= & \frac{c}{f_{12}^{2} f_{23}^{2} f_{13}^{2}}, \\
\left\langle T\left(f_{1}\right) T\left(f_{2}\right) \mathcal{A}\left(f_{3}\right)\right\rangle_{C}= & \frac{c(5 c+22)}{10} \frac{1}{f_{23}^{4} f_{13}^{4}}, \\
\left\langle T\left(f_{1}\right) T\left(f_{2}\right) T\left(f_{3}\right) T\left(f_{4}\right)\right\rangle_{C}= & c\left(\frac{1}{\left(f_{12} f_{34} f_{13} f_{24}\right)^{2}}+\frac{1}{\left(f_{13} f_{24} f_{14} f_{23}\right)^{2}}+\frac{1}{\left(f_{12} f_{34} f_{14} f_{23}\right)^{2}}\right) \\
& +\frac{c^{2}}{4}\left(\frac{1}{\left(f_{12} f_{34}\right)^{4}}+\frac{1}{\left(f_{13} f_{24}\right)^{4}}+\frac{1}{\left(f_{14} f_{23}\right)^{4}}\right),
\end{aligned}
$$


where $f_{i j} \equiv f_{i}-f_{j}$. And then we get

$$
\begin{aligned}
d_{T T T}^{j_{1} j_{2} j_{3}}= & -\frac{1}{8 n^{6} c^{2}} \frac{1}{s_{j_{1} j_{2}}^{2} s_{j_{2} j_{3}}^{2} s_{j_{3} j_{1}}^{2}}+\frac{n^{2}-1}{96 n^{6} c}\left(\frac{1}{s_{j_{1} j_{2}}^{4}}+\frac{1}{s_{j_{2} j_{3}}^{4}}+\frac{1}{s_{j_{3} j_{1}}^{4}}\right)+\frac{\left(n^{2}-1\right)^{3}}{1728 n^{6}}, \\
d_{T T \mathcal{A}}^{j_{1} j_{2} j_{3}}= & \frac{1}{64 n^{8} c^{2}} \frac{1}{\left(s_{j_{1} j_{3}} s_{j_{2} j_{3}}\right)^{4}}-\frac{n^{2}-1}{96 n^{8} c^{2}} \frac{1}{\left(s_{j_{1} j_{2}} s_{j_{1} j_{3}} s_{\left.j_{2} j_{3}\right)^{2}}\right.} \\
& +\frac{\left(n^{2}-1\right)^{2}}{2304 n^{8} c}\left(\frac{1}{s_{j_{1} j_{2}}^{4}}+\frac{2}{s_{j_{1} j_{3}}^{4}}+\frac{2}{s_{j_{2} j_{3}}^{4}}\right)+\frac{\left(n^{2}-1\right)^{4}}{41472 n^{8}}, \\
d_{T T T T}^{j_{1} j_{2} j_{3} j_{4}}= & \frac{1}{16 n^{8} c^{3}} g_{\alpha}^{j_{1} j_{2} j_{3} j_{4}}+\frac{1}{64 n^{8} c^{2}} g_{\beta}^{j_{1} j_{2} j_{3} j_{4}}-\frac{n^{2}-1}{96 n^{8} c^{2}} g_{\gamma}^{j_{1} j_{2} j_{3} j_{4}}+\frac{\left(n^{2}-1\right)^{2}}{1152 n^{8} c} g_{\delta}^{j_{1} j_{2} j_{3} j_{4}}+\frac{\left(n^{2}-1\right)^{4}}{20736 n^{8}},
\end{aligned}
$$

where

$$
\begin{aligned}
& g_{\alpha}^{j_{1} j_{2} j_{3} j_{4}}=\frac{1}{\left(s_{j_{1} j_{2}} s_{j_{3} j_{4}} s_{j_{1} j_{3}} s_{j_{2} j_{4}}\right)^{2}}+\frac{1}{\left(s_{j_{1} j_{3}} s_{j_{2} j_{4}} s_{j_{1} j_{4}} s_{j_{2} j_{3}}\right)^{2}}+\frac{1}{\left(s_{j_{1} j_{2}} s_{j_{3} j_{4}} s_{j_{1} j_{4}} s_{j_{2} j_{3}}\right)^{2}}, \\
& g_{\beta}^{j_{1} j_{2} j_{3} j_{4}}=\frac{1}{\left(s_{j_{1} j_{2}} s_{j_{3} j_{4}}\right)^{4}}+\frac{1}{\left(s_{j_{1} j_{3}} s_{j_{2} j_{4}}\right)^{4}}+\frac{1}{\left(s_{j_{1} j_{4}} s_{j_{2} j_{3}}\right)^{4}}, \\
& g_{\gamma}^{j_{1} j_{2} j_{3} j_{4}}=\frac{1}{\left(s_{j_{1} j_{2}} s_{j_{2} j_{3}} s_{j_{3} j_{1}}\right)^{2}}+\frac{1}{\left(s_{j_{1} j_{2}} s_{j_{2} j_{4}} s_{j_{4} j_{1}}\right)^{2}}+\frac{1}{\left(s_{j_{1} j_{3}} s_{j_{3} j_{4}} s_{j_{4} j_{1}}\right)^{2}}+\frac{1}{\left(s_{j_{2} j_{3}} s_{j_{3} j_{4}} s_{j_{4} j_{2}}\right)^{2}} \text {, } \\
& g_{\delta}^{j_{1} j_{2} j_{3} j_{4}}=\frac{1}{s_{j_{1} j_{2}}^{4}}+\frac{1}{s_{j_{1} j_{3}}^{4}}+\frac{1}{s_{j_{1} j_{4}}^{4}}+\frac{1}{s_{j_{2} j_{3}}^{4}}+\frac{1}{s_{j_{2} j_{4}}^{4}}+\frac{1}{s_{j_{3} j_{4}}^{4}} \text {. }
\end{aligned}
$$

There are other operators that cannot be factorized, and the coefficients $d_{K}$ for these operators are

$$
\begin{aligned}
& d_{\mathcal{J}}^{j_{1} j_{2}}=\frac{1}{16 n^{5} c} \frac{c_{j_{1} j_{2}}}{s_{j_{1} j_{2}}^{5}}, \quad d_{\mathcal{K}}^{j_{1} j_{2}}=\frac{5}{128 n^{6} c} \frac{1}{s_{j_{1} j_{2}}^{6}}-\frac{n^{2}+9}{288 n^{6} c} \frac{1}{s_{j_{1} j_{2}}^{4}}-\frac{\left(n^{2}-1\right)^{2}}{5184 n^{4}}, \\
& d_{\mathcal{L}}^{j_{1} j_{2}}=\frac{n^{2}-1}{384 n^{7} c} \frac{c_{j_{1} j_{2}}}{s_{j_{1} j_{2}}^{5}}, \quad d_{\mathcal{M}}^{j_{1} j_{2}}=\frac{3}{256 n^{7} c} \frac{c_{j_{1} j_{2}}}{s_{j_{1} j_{2}}^{7}}-\frac{4 n^{2}+11}{1760 n^{7} c} \frac{c_{j_{1} j_{2}}}{s_{j_{1} j_{2}}^{5}}, \\
& d_{T \mathcal{J}}^{j_{1} j_{2} j_{3}}=-\frac{1}{64 n^{7} c^{2}} \frac{2 c_{j_{2} j_{3}} s_{j_{1} j_{2}} s_{j_{1} j_{3}}-s_{j_{2} j_{3}}^{2}}{\left(s_{j_{1} j_{2}} s_{j_{1} j_{3}} s_{j_{2} j_{3}}\right)^{3}}-\frac{n^{2}-1}{384 n^{7} c}\left(\frac{c_{j_{1} j_{2}}}{s_{j_{1} j_{2}}^{5}}-\frac{c_{j_{1} j_{3}}}{s_{j_{1} j_{3}}^{5}}-\frac{2 c_{j_{2} j_{3}}}{s_{j_{2} j_{3}}^{5}}\right), \\
& d_{\mathcal{N}}^{j_{1} j_{2} j_{3}}=\frac{1}{3}\left(d_{T \mathcal{J}}^{j_{1} j_{2} j_{3}}+2 d_{T \mathcal{J}}^{j_{3} j_{1} j_{2}}\right), \quad d_{\mathcal{O}}^{j_{1} j_{2}}=\frac{n^{2}-1}{39936 n^{8} c} \frac{65-4\left(2 n^{2}+13\right) s_{j_{1} j_{2}}^{2}}{s_{j_{1} j_{2}}^{6}}-\frac{\left(n^{2}-1\right)^{3}}{179712 n^{6}}, \\
& d_{\mathcal{P}}^{j_{1} j_{2}}=\frac{1}{7321600 n^{8} c} \frac{30030-220\left(41 n^{2}+169\right) s_{j_{1} j_{2}}^{2}+16\left(27 n^{4}+451 n^{2}+572\right) s_{j_{1} j_{2}}^{4}}{s_{j_{1} j_{2}}^{8}}+\frac{3\left(n^{2}-1\right)^{2}}{915200 n^{4}}, \\
& d_{T \mathcal{K}}^{j_{1} j_{2} j_{3}}=-\frac{1}{2304 n^{8} c^{2}} \frac{14 s_{j_{2} j_{3}}^{4}+27 s_{j_{1} j_{2}}^{2} s_{j_{1} j_{3}}^{2}-8 s_{j_{2} j_{3}}^{2}\left(s_{j_{1} j_{2}}^{2}+s_{j_{1} j_{3}}^{2}\right)-8\left(n^{2}+2\right)\left(s_{j_{1} j_{2}} s_{j_{1} j_{3}} s_{j_{2} j_{3}}\right)^{2}}{\left(s_{j_{1} j_{2}} s_{j_{1} j_{3}} s_{j_{2} j_{3}}\right)^{4}} \\
& +\frac{n^{2}-1}{6912 n^{8} c}\left(\frac{5-2\left(n^{2}+2\right) s_{j_{1} j_{2}}^{2}}{s_{j_{1} j_{2}}^{6}}+\frac{5-2\left(n^{2}+2\right) s_{j_{1} j_{3}}^{3}}{s_{j_{1} j_{3}}^{6}}+\frac{45-4\left(n^{2}+9\right) s_{j_{2} j_{3}}^{2}}{2 s_{j_{2} j_{3}}^{6}}\right) \\
& -\frac{\left(n^{2}-1\right)^{3}}{62208 n^{6}} \\
& d_{\mathcal{Q}}^{j_{1} j_{2} j_{3}}=\frac{81}{77}\left(d_{T \mathcal{K}}^{j_{2} j_{3} j_{1}}-\frac{2}{9} d_{T \mathcal{K}}^{j_{1} j_{2} j_{3}}\right), \quad d_{\mathcal{R}}^{j_{1} j_{2} j_{3}}=\frac{99}{91}\left(d_{T \mathcal{K}}^{j_{3} j_{1} j_{2}}-\frac{2}{11}\left(d_{T \mathcal{K}}^{j_{1} j_{2} j_{3}}+d_{T \mathcal{K}}^{j_{2} j_{3} j_{1}}\right)\right) .
\end{aligned}
$$

Here $c_{j_{1} j_{2}} \equiv \cos \frac{\pi\left(j_{1}-j_{2}\right)}{n}$. 
In the presence of $W_{3}$ symmetry, the additional quasiprimary operators listed in the table above (A.20) have the coefficients

$$
\begin{aligned}
& d_{W W}^{j_{1} j_{2}}=-\frac{3}{(2 n)^{6} c} \frac{1}{s_{j_{1} j_{2}}^{6}}, \quad d_{\mathcal{U}}^{j_{1} j_{2}}=-\frac{3}{(2 n)^{7} c} \frac{c_{j_{1} j_{2}}}{s_{j_{1} j_{2}}^{7}}, \quad d_{W \mathcal{S}}^{j_{1} j_{2}}=-\frac{n^{2}-1}{(2 n)^{8} c} \frac{1}{s_{j_{1} j_{2}}^{6}}, \\
& d_{\mathcal{V}}^{j_{1} j_{2}}=\frac{1}{26(2 n)^{8} c}\left(\frac{6\left(n^{2}+13\right)}{s_{j_{1} j_{2}}^{6}}-\frac{91}{s_{j_{1} j_{2}}^{8}}\right), \quad d_{T W W}^{j_{1} j_{2} j_{3}}=\frac{18}{(2 n)^{8} c^{2}} \frac{1}{s_{j_{1} j_{2}}^{2} s_{j_{2} j_{3}}^{4} s_{j_{3} j_{1}}^{2}}-\frac{n^{2}-1}{(2 n)^{8} c} \frac{1}{s_{j_{2} j_{3}}^{6}} .
\end{aligned}
$$

In the calculation we need to use the three-point function

$$
\left\langle T\left(f_{1}\right) W\left(f_{2}\right) W\left(f_{3}\right)\right\rangle_{C}=\frac{c}{f_{12}^{2} f_{13}^{2} f_{23}^{4}} .
$$

In the presence of $W_{4}$ symmetry we have

$$
d_{W_{4} W_{4}}^{j_{1} j_{2}}=\frac{1}{(2 n)^{8}} \frac{1}{s_{j_{1} j_{2}}^{8}} .
$$

\section{Some details in subsection 3.2}

It is useful to find that

$$
\begin{aligned}
& \alpha_{T \mathcal{J}}\left(d_{T \mathcal{J}}^{j_{1} j_{2} j_{3}}\right)^{2}+\alpha_{\mathcal{N}}\left(d_{\mathcal{N}}^{j_{1} j_{2} j_{3}}\right)^{2}=\frac{2 c^{3}}{3}\left(\left(d_{T \mathcal{J}}^{j_{1} j_{2} j_{3}}\right)^{2}+\left(d_{T \mathcal{J}}^{j_{2} j_{3} j_{1}}\right)^{2}+\left(d_{T \mathcal{J}}^{j_{3} j_{1} j_{2}}\right)^{2}\right) \\
&= \frac{4}{(2 n)^{14} c}\left(h_{331}^{j_{1} j_{2} j_{3}}+2 h_{322}^{j_{1} j_{2} j_{3}}-12 h_{222}^{j_{1} j_{2} j_{3}}\right)-\frac{8\left(n^{2}-1\right)}{3(2 n)^{14}} h_{\alpha}^{j_{1} j_{2} j_{3}} \\
& \quad+\frac{4\left(n^{2}-1\right)^{2} c}{9(2 n)^{14}}\left(h_{500}^{j_{1} j_{2} j_{3}}-h_{400}^{j_{1} j_{2} j_{3}}-h_{\beta}^{j_{1} j_{2} j_{3}}\right) .
\end{aligned}
$$

Here we have

$$
\begin{gathered}
h_{\alpha}^{j_{1} j_{2} j_{3}}=\frac{1}{\left(s_{j_{1} j_{2}} s_{j_{2} j_{3}} s_{j_{3} j_{1}}\right)^{3}}\left(\frac{c_{j_{1} j_{2}}}{s_{j_{1} j_{2}}^{5}}\left(2 s_{j_{1} j_{2}}^{2}-s_{j_{2} j_{3}}^{2}-s_{j_{3} j_{1}}^{2}\right)+\frac{c_{j_{2} j_{3}}}{s_{j_{2} j_{3}}^{5}}\left(2 s_{j_{2} j_{3}}^{2}-s_{j_{3} j_{1}}^{2}-s_{j_{1} j_{2}}^{2}\right)\right. \\
\left.\quad+\frac{c_{j_{3} j_{1}}}{s_{j_{3} j_{1}}^{5}}\left(2 s_{j_{3} j_{1}}^{2}-s_{j_{1} j_{2}}^{2}-s_{j_{2} j_{3}}^{2}\right)\right) \\
h_{\beta}^{j_{1} j_{2} j_{3}}=\frac{c_{j_{1} j_{2}} c_{j_{2} j_{3}}}{s_{j_{1} j_{2}}^{5} s_{j_{2} j_{3}}^{5}}+\frac{c_{j_{2} j_{3}} c_{j_{3} j_{1}}}{s_{j_{2} j_{3}}^{5} s_{j_{3} j_{1}}^{5}}+\frac{c_{j_{3} j_{1}} c_{j_{1} j_{2}}}{s_{j_{3} j_{1}}^{5} s_{j_{1} j_{2}}^{5}}
\end{gathered}
$$

and

$$
h_{m p q}^{j_{1} j_{2} j_{3}}=\frac{1}{s_{j_{1} j_{2}}^{2 m} s_{j_{2} j_{3}}^{2 p} s_{j_{3} j_{1}}^{2 q}}+c y c .
$$

with the indexes $m p q$ being totally symmetric and $c y c$. being some possible cyclic terms. For examples, there is

$$
h_{m m m}^{j_{1} j_{2} j_{3}}=\frac{1}{s_{j_{1} j_{2}}^{2 m} s_{j_{2} j_{3}}^{2 m} s_{j_{3} j_{1}}^{2 m}},
$$

and for $m \neq p$ there is

$$
h_{m m p}^{j_{1} j_{2} j_{3}}=\frac{1}{s_{j_{1} j_{2}}^{2 m} s_{j_{2} j_{3}}^{2 m} s_{j_{3} j_{1}}^{2 p}}+\frac{1}{s_{j_{1} j_{2}}^{2 m} s_{j_{2} j_{3}}^{2 p} s_{j_{3} j_{1}}^{2 m}}+\frac{1}{s_{j_{1} j_{2}}^{2 p} s_{j_{2} j_{3}}^{2 m} s_{j_{3} j_{1}}^{2 m}}
$$


and for $m \neq p, m \neq q, p \neq q$ there is

$h_{m p q}^{j_{1} j_{2} j_{3}}=\frac{1}{s_{j_{1} j_{2}}^{2 m} s_{j_{2} j_{3}}^{2 p} s_{j_{3} j_{1}}^{2 q}}+\frac{1}{s_{j_{1} j_{2}}^{2 m} s_{j_{2} j_{3}}^{2 q} s_{j_{3} j_{1}}^{2 p}}+\frac{1}{s_{j_{1} j_{2}}^{2 q} s_{j_{2} j_{3}}^{2 m} s_{j_{3} j_{1}}^{2 p}}+\frac{1}{s_{j_{1} j_{2}}^{2 p} s_{j_{2} j_{3}}^{2 m} s_{j_{3} j_{1}}^{2 q}}+\frac{1}{s_{j_{1} j_{2}}^{2 p} s_{j_{2} j_{3}}^{2 q} s_{j_{3} j_{1}}^{2 m}}+\frac{1}{s_{j_{1} j_{2}}^{2 q} s_{j_{2} j_{3}}^{2 p} s_{j_{3} j_{1}}^{2 m}}$.

Another useful relation is

$$
\begin{aligned}
& \alpha_{T \mathcal{K}}\left(d_{T \mathcal{K}}^{j_{1} j_{2} j_{3}}\right)^{2}+\alpha_{\mathcal{Q}}\left(d_{\mathcal{Q}}^{j_{1} j_{2} j_{3}}\right)^{2}+\alpha_{\mathcal{R}}\left(d_{\mathcal{R}}^{j_{1} j_{2} j_{3}}\right)^{2} \\
= & \frac{163 c^{3}}{455}\left(11\left(\left(d_{T \mathcal{J}}^{j_{1} j_{2} j_{3}}\right)^{2}+\left(d_{T \mathcal{J}}^{j_{2} j_{3} j_{1}}\right)^{2}+\left(d_{T \mathcal{J}}^{j_{3} j_{1} j_{2}}\right)^{2}\right)\right. \\
& \left.\quad-4\left(d_{T \mathcal{J}}^{j_{1} j_{2} j_{3}} d_{T \mathcal{J}}^{j_{2} j_{3} j_{1}}+d_{T \mathcal{J}}^{j_{2} j_{3} j_{1}} d_{T \mathcal{J}}^{j_{3} j_{1} j_{2}}+d_{T \mathcal{J}}^{j_{3} j_{1} j_{2}} d_{T \mathcal{J}}^{j_{1} j_{2} j_{3}}\right)\right) \\
= & \frac{1}{2129920 n^{16} c} I-\frac{n^{2}-1}{6389760 n^{16}} I I+\frac{\left(n^{2}-1\right)^{2} c}{230031360 n^{16}} I I I-\frac{\left(n^{2}-1\right)^{4} c^{2}}{172523520 n^{14}} I V+\frac{\left(n^{2}-1\right)^{6} c^{3}}{517570560 n^{12}},
\end{aligned}
$$

where

$$
\begin{aligned}
I= & 308 h_{440}^{j_{1} j_{2} j_{3}}+1445 h_{422}^{j_{1} j_{2} j_{3}}-120 h_{332}^{j_{1} j_{2} j_{3}}-504 h_{431}^{j_{1} j_{2} j_{3}}-16\left(n^{2}+2\right)\left(14 h_{331}^{j_{1} j_{2} j_{3}}+11 h_{322}^{j_{1} j_{2} j_{3}}\right) \\
& +192\left(n^{2}+2\right)^{2} h_{222}^{j_{1} j_{2} j_{3}} \\
I I= & 1755 h_{511}^{j_{1} j_{2} j_{3}}+910 h_{322}^{j_{1} j_{2} j_{3}}-520 h_{421}^{j_{1} j_{2} j_{3}}-168\left(n^{2}+13\right) h_{222}^{j_{1} j_{2} j_{3}}-188\left(3 n^{2}+13\right) h_{411}^{j_{1} j_{2} j_{3}} \\
& -4\left(11 n^{2}-104\right) h_{321}^{j_{1} j_{2} j_{3}}-56 n^{2} h_{420}^{j_{1} j_{2} j_{3}}+32\left(n^{2}+2\right)\left(3 n^{2}+13\right) h_{311}^{j_{1} j_{2} j_{3}}, \\
I I I= & 8775 h_{600}^{j_{1} j_{2} j_{3}}+3900 h_{330}^{j_{1} j_{2} j_{3}}-1560\left(n^{2}+9\right) h_{500}^{j_{1} j_{2} j_{3}}-1560\left(n^{2}+2\right) h_{320}^{j_{1} j_{2} j_{3}} \\
& +48\left(3 n^{4}+26 n^{2}+117\right) h_{400}^{j_{1} j_{2} j_{3}}+16\left(25 n^{4}+149 n^{2}+156\right) h_{220}^{j_{1} j_{2} j_{3}} \\
& +88 n^{2}\left(n^{2}-1\right) h_{211}^{j_{1} j_{2} j_{3}}-192 n^{2}\left(n^{2}-1\right)\left(n^{2}+2\right) h_{111}^{j_{1} j_{2} j_{3}}, \\
I V= & 65 h_{300}^{j_{1} j_{2} j_{3}}-4\left(3 n^{2}+13\right) h_{200}^{j_{1} j_{2} j_{3}} .
\end{aligned}
$$

\section{One short interval on cylinder}

This appendix is a byproduct of the paper. Here we compute the Rényi entropy for the case of a short interval with length $\ell$ on a cylinder. We choose the spatial part of the 2D CFT is a circle of length $L$. The Rényi entanglement entropy of $A$ is known [7]

$$
S_{n}=\frac{c}{6}\left(1+\frac{1}{n}\right) \log \left(\frac{L}{\pi \epsilon} \sin \frac{\pi \ell}{L}\right) .
$$

Using the OPE of twist operators, the result was reproduced to order $O\left(\ell^{6} / L^{6}\right)$ in [3], and the result in this paper allows us to calculate to order $O\left(\ell^{8} / L^{8}\right)$.

It is shown that in [3] that the Rényi entropy is

$$
\operatorname{Tr} \rho_{A}^{n}=c_{n} \ell^{-\frac{c}{6}\left(n-\frac{1}{n}\right)}\left(\sum_{K} d_{K} \ell^{h_{K}}\left\langle\Phi_{K}(0)\right\rangle_{L}\right)^{2},
$$

with $K$ being the summation over all the linear independent holomorphic quasiprimary operators constructed solely by the operators in the Virasoro Verma module. 
With the transformation formulas in the previous section we could get

$$
\begin{array}{rlrl}
\langle T(0)\rangle_{L} & =\frac{\pi^{2} c}{6 L^{2}}, \quad\langle\mathcal{A}(0)\rangle_{L}=\frac{\pi^{4} c(5 c+22)}{180 L^{4}}, & \langle\mathcal{B}(0)\rangle_{L}=-\frac{62 \pi^{6} c}{525 L^{6}} \\
\langle\mathcal{D}(0)\rangle_{L} & =\frac{\pi^{6} c(2 c-1)(5 c+22)(7 c+68)}{216(70 c+29) L^{6}}, & \langle\mathcal{E}(0)\rangle_{L}=\frac{23452 \pi^{8} c}{59535 L^{8}} \\
\langle\mathcal{H}(0)\rangle_{L} & =-\frac{13 \pi^{8} c(5 c+22)(465 c-127)}{10125(105 c+11) L^{8}}, & \\
\langle\mathcal{I}(0)\rangle_{L} & =\frac{\pi^{8} c(2 c-1)(3 c+46)(5 c+3)(5 c+22)(7 c+68)}{1296(5 c(210 c+661)-251) L^{8}}
\end{array}
$$

Then we could find the Rényi entanglement entropy

$$
\begin{aligned}
S_{n} & =-\frac{1}{n-1} \log \operatorname{Tr} \rho_{A}^{n} \\
& =\frac{c}{6}\left(1+\frac{1}{n}\right)\left(\log \frac{\ell}{\epsilon}-\frac{\pi^{2} \ell^{2}}{6 L^{2}}-\frac{\pi^{4} \ell^{4}}{180 L^{4}}-\frac{\pi^{6} \ell^{6}}{2835 L^{6}}-\frac{\pi^{8} \ell^{8}}{37800 L^{8}}+O\left(\frac{\ell^{10}}{L^{10}}\right)\right),
\end{aligned}
$$

which matches (D.1) to the order of $O\left(\ell^{8} / L^{8}\right)$.

The finite temperature effect is the same with the finite length case if we substitute $L \rightarrow i \beta$ with $\beta$ being the inverse temperature.

\section{E Some useful summation formulas}

In this appendix we summarize some formulas that are needed in our calculation. We define

$$
f_{m}=\sum_{j=1}^{n-1} \frac{1}{\left(\sin \frac{\pi j}{n}\right)^{2 m}} .
$$

We have also defined $h_{\alpha}^{j_{1} j_{2} j_{3}}, h_{\beta}^{j_{1} j_{2} j_{3}}$ in (C.2), $h_{m p q}^{j_{1} j_{2} j_{3}}$ in (C.3), and $g_{\alpha}^{j_{1} j_{2} j_{3} j_{4}}, g_{\beta}^{j_{1} j_{2} j_{3} j_{4}}, g_{\gamma}^{j_{1} j_{2} j_{3} j_{4}}$, $g_{\delta}^{j_{1} j_{2} j_{3} j_{4}}$ in (B.11).

Explicitly we need

$$
\begin{aligned}
f_{1}= & \frac{n^{2}-1}{3}, \quad f_{2}=\frac{\left(n^{2}-1\right)\left(n^{2}+11\right)}{45}, \quad f_{3}=\frac{\left(n^{2}-1\right)\left(2 n^{4}+23 n^{2}+191\right)}{945} \\
f_{4}= & \frac{\left(n^{2}-1\right)\left(n^{2}+11\right)\left(3 n^{4}+10 n^{2}+227\right)}{14175}, \\
f_{5}= & \frac{\left(n^{2}-1\right)\left(2 n^{8}+35 n^{6}+321 n^{4}+2125 n^{2}+14797\right)}{93555} \\
f_{6}= & \frac{\left(n^{2}-1\right)\left(1382 n^{10}+28682 n^{8}+307961 n^{6}+2295661 n^{4}+13803157 n^{2}+92427157\right)}{638512875} \\
f_{7}= & \frac{\left(n^{2}-1\right)\left(60 n^{12}+1442 n^{10}+17822 n^{8}+151241 n^{6}+997801 n^{4}+5636617 n^{2}+36740617\right)}{273648375} \\
f_{8}= & \frac{\left(n^{2}-1\right)}{488462349375}\left(10851 n^{14}+296451 n^{12}+4149467 n^{10}+39686267 n^{8}+292184513 n^{6}\right. \\
& \left.+1777658113 n^{4}+9611679169 n^{2}+61430943169\right)
\end{aligned}
$$


The above formulas are useful because it often appears in the calculation that

$$
\begin{gathered}
\sum_{0 \leq j_{1}<j_{2} \leq n-1} \frac{1}{s_{j_{1} j_{2}}^{2 m}}=\frac{n}{2} f_{m}, \\
\sum_{0 \leq j_{1}<j_{2}<j_{3} \leq n-1} h_{m 00}^{j_{1} j_{2} j_{3}}=\frac{n(n-2)}{2} f_{m} .
\end{gathered}
$$

There are also several summation formulas listed below.

$$
\begin{aligned}
& \sum_{0 \leq j_{1}<j_{2}<j_{3} \leq n-1} h_{\alpha}^{j_{1} j_{2} j_{3}}=\frac{2 n\left(n^{2}-1\right)\left(n^{2}-4\right)\left(n^{2}-9\right)\left(319 n^{6}+13566 n^{4}+152271 n^{2}+892244\right)}{638512875}, \\
& \sum_{0 \leq j_{1}<j_{2}<j_{3} \leq n-1} h_{\beta}^{j_{1} j_{2} j_{3}}=\frac{n\left(n^{2}-1\right)\left(n^{2}-4\right)\left(5 n^{6}+58 n^{4}+325 n^{2}+1052\right)}{467775}, \\
& \sum_{0 \leq j_{1}<j_{2}<j_{3} \leq n-1} h_{111}^{j_{1} j_{2} j_{3}}=\frac{n\left(n^{2}-1\right)\left(n^{2}-4\right)\left(n^{2}+47\right)}{2835}, \\
& \sum_{0 \leq j_{1}<j_{2}<j_{3} \leq n-1} h_{211}^{j_{1} j_{2} j_{3}}=\frac{n\left(n^{2}-1\right)\left(n^{2}-4\right)\left(n^{4}+40 n^{2}+679\right)}{14175}, \\
& \sum_{0 \leq j_{1}<j_{2}<j_{3} \leq n-1} h_{220}^{j_{1} j_{2} j_{3}}=\frac{2 n\left(n^{2}-1\right)\left(n^{2}-4\right)\left(n^{2}+11\right)\left(n^{2}+19\right)}{14175}, \\
& \sum_{0 \leq j_{1}<j_{2}<j_{3} \leq n-1} h_{320}^{j_{1} j_{2} j_{3}}=\frac{2 n\left(n^{2}-1\right)\left(n^{2}-4\right)\left(6 n^{6}+173 n^{4}+2084 n^{2}+12137\right)}{467775}, \\
& \sum_{0 \leq j_{1}<j_{2}<j_{3} \leq n-1} h_{330}^{j_{1} j_{2} j_{3}}=\frac{n\left(n^{2}-1\right)\left(n^{2}-4\right)}{638512875}\left(739 n^{8}+20075 n^{6}+355677 n^{4}\right. \\
& \left.+2953625 n^{2}+14813884\right) \\
& \sum_{0 \leq j_{1}<j_{2}<j_{3} \leq n-1} h_{311}^{j_{1} j_{2} j_{3}}=\frac{n\left(n^{2}-1\right)\left(n^{2}-4\right)\left(3 n^{6}+125 n^{4}+1757 n^{2}+21155\right)}{467775} \\
& \sum_{0 \leq j_{1}<j_{2}<j_{3} \leq n-1} h_{222}^{j_{1} j_{2} j_{3}}=\frac{n\left(n^{2}-1\right)\left(n^{2}-4\right)\left(19 n^{8}+875 n^{6}+22317 n^{4}+505625 n^{2}+5691964\right)}{273648375}, \\
& \sum_{0 \leq j_{1}<j_{2}<j_{3} \leq n-1} h_{321}^{j_{1} j_{2} j_{3}}=\frac{n\left(n^{2}-1\right)\left(n^{2}-4\right)}{638512875}\left(473 n^{8}+18745 n^{6}+458199 n^{4}\right. \\
& \left.+6674755 n^{2}+65423828\right) \\
& \sum_{0 \leq j_{1}<j_{2}<j_{3} \leq n-1} h_{420}^{j_{1} j_{2} j_{3}}=\frac{n\left(n^{2}-1\right)\left(n^{2}-4\right)}{638512875}\left(1621 n^{8}+50875 n^{6}+630273 n^{4}\right. \\
& \left.+5624825 n^{2}+29980406\right) \\
& \sum_{0 \leq j_{1}<j_{2}<j_{3} \leq n-1} h_{411}^{j_{1} j_{2} j_{3}}=\frac{n\left(n^{2}-1\right)\left(n^{2}-4\right)}{638512875}\left(404 n^{8}+17945 n^{6}+276297 n^{4}\right. \\
& \left.+2703955 n^{2}+27241399\right),
\end{aligned}
$$




$$
\begin{aligned}
& \sum_{0 \leq j_{1}<j_{2}<j_{3} \leq n-1} h_{421}^{j_{1} j_{2} j_{3}}=\frac{n\left(n^{2}-1\right)\left(n^{2}-4\right)}{1915538625}\left(138 n^{10}+5819 n^{8}+146479 n^{6}+2091357 n^{4}\right. \\
& \left.+22440283 n^{2}+193043924\right) \\
& \sum_{0 \leq j_{1}<j_{2}<j_{3} \leq n-1} h_{511}^{j_{1} j_{2} j_{3}}=\frac{n\left(n^{2}-1\right)\left(n^{2}-4\right)}{1915538625}\left(122 n^{10}+5798 n^{8}+100301 n^{6}+1060609 n^{4}\right. \\
& \left.+8570077 n^{2}+77354293\right) \\
& \sum_{0 \leq j_{1}<j_{2}<j_{3} \leq n-1} h_{322}^{j_{1} j_{2} j_{3}}=\frac{n\left(n^{2}-1\right)\left(n^{2}-4\right)}{383107725}\left(6 n^{10}+299 n^{8}+8023 n^{6}+168477 n^{4}\right. \\
& \left.+2635831 n^{2}+26217764\right) \\
& \sum_{0 \leq j_{1}<j_{2}<j_{3} \leq n-1} h_{331}^{j_{1} j_{2} j_{3}}=\frac{2 n\left(n^{2}-1\right)\left(n^{2}-4\right)}{1915538625}\left(30 n^{10}+1229 n^{8}+27865 n^{6}+529947 n^{4}\right. \\
& \left.+6100405 n^{2}+51401324\right) \\
& \sum_{0 \leq j_{1}<j_{2}<j_{3} \leq n-1} h_{431}^{j_{1} j_{2} j_{3}}=\frac{2 n\left(n^{2}-1\right)\left(n^{2}-4\right)}{488462349375}\left(1477 n^{12}+63995 n^{10}+1496434 n^{8}+27120610 n^{6}\right. \\
& \left.+367070101 n^{4}+3500775395 n^{2}+26585391988\right), \\
& \sum_{0 \leq j_{1}<j_{2}<j_{3} \leq n-1} h_{332}^{j_{1} j_{2} j_{3}}=\frac{4 n\left(n^{2}-1\right)\left(n^{2}-4\right)}{488462349375}\left(128 n^{12}+7015 n^{10}+203101 n^{8}+4225095 n^{6}\right. \\
& \left.+78482039 n^{4}+1054880390 n^{2}+9748602232\right), \\
& \sum_{0 \leq j_{1}<j_{2}<j_{3} \leq n-1} h_{422}^{j_{1} j_{2} j_{3}}=\frac{n\left(n^{2}-1\right)\left(n^{2}-4\right)}{488462349375}\left(709 n^{12}+37205 n^{10}+1040278 n^{8}+22228690 n^{6}\right. \\
& \left.+325794217 n^{4}+3892862105 n^{2}+34949076796\right), \\
& \sum_{0 \leq j_{1}<j_{2}<j_{3} \leq n-1} h_{440}^{j_{1} j_{2} j_{3}}=\frac{2 n\left(n^{2}-1\right)\left(n^{2}-4\right)}{488462349375}\left(2757 n^{12}+88245 n^{10}+1511414 n^{8}+20490610 n^{6}\right. \\
& \left.+181728201 n^{4}+1105797145 n^{2}+4786765628\right),
\end{aligned}
$$

There are also the summations of four indexes.

$$
\begin{aligned}
\sum_{0 \leq j_{1}<j_{2}<j_{3}<j_{4} \leq n-1} g_{\alpha}^{j_{1} j_{2} j_{3} j_{4}} & =\frac{n\left(n^{2}-1\right)\left(n^{2}-4\right)\left(n^{2}-9\right)\left(n^{2}+119\right)}{28350}, \\
\sum_{0 \leq j_{1}<j_{2}<j_{3}<j_{4} \leq n-1} g_{\beta}^{j_{1} j_{2} j_{3} j_{4}} & =\frac{n(n-2)(n-3)\left(n^{2}-1\right)\left(n^{2}+11\right)\left(7 n^{3}+13 n^{2}+93 n+127\right)}{113400}, \\
\sum_{0 \leq j_{1}<j_{2}<j_{3}<j_{4} \leq n-1} g_{\gamma}^{j_{1} j_{2} j_{3} j_{4}} & =\frac{n(n-3)\left(n^{2}-1\right)\left(n^{2}-4\right)\left(n^{2}+47\right)}{2835},
\end{aligned}
$$




$$
\begin{aligned}
\sum_{0 \leq j_{1}<j_{2}<j_{3}<j_{4} \leq n-1} g_{\delta}^{j_{1} j_{2} j_{3} j_{4}}=\frac{n(n-2)(n-3)\left(n^{2}-1\right)\left(n^{2}+11\right)}{180} & \\
\sum_{0 \leq j_{1}<j_{2}<j_{3}<j_{4} \leq n-1}\left(g_{\alpha}^{j_{1} j_{2} j_{3} j_{4}}\right)^{2}= & \frac{n\left(n^{2}-1\right)\left(n^{2}-4\right)\left(n^{2}-9\right)}{54273594375}\left(21 n^{10}+1994 n^{8}+105648 n^{6}\right. \\
& \left.+4785522 n^{4}+141534331 n^{2}+2127620484\right) \\
\sum_{0 \leq j_{1}<j_{2}<j_{3}<j_{4} \leq n-1}\left(g_{\beta}^{j_{1} j_{2} j_{3} j_{4}}\right)^{2}= & \frac{n(n-2)(n-3)\left(n^{2}-1\right)}{3907698795000}\left(21879 n^{13}+45093 n^{12}+699510 n^{11}\right. \\
& +1522530 n^{10}+12198793 n^{9}+30819611 n^{8}+178371380 n^{7} \\
& +647286940 n^{6}+2857453977 n^{5}+14207989899 n^{4} \\
& +57188421110 n^{3}+224193314530 n^{2}+788902033351 n \\
& +949713901397)
\end{aligned}
$$$$
\sum_{0 \leq j_{1}<j_{2}<j_{3}<j_{4} \leq n-1}\left(g_{\gamma}^{j_{1} j_{2} j_{3} j_{4}}\right)^{2}=\frac{n(n-3)\left(n^{2}-1\right)\left(n^{2}-4\right)}{1915538625}\left(133 n^{8}+519 n^{7}+7682 n^{6}+45486 n^{5}\right.
$$$$
\left.+292677 n^{4}+1470231 n^{3}+7950068 n^{2}+35678964 n+146880640\right),
$$$$
\sum_{0 \leq j_{1}<j_{2}<j_{3}<j_{4} \leq n-1}\left(g_{\delta}^{j_{1} j_{2} j_{3} j_{4}}\right)^{2}=\frac{n(n-2)(n-3)\left(n^{2}-1\right)\left(n^{2}+11\right)}{56700}\left(3 n^{4}+23 n^{3}\right.
$$$$
\left.+55 n^{2}+397 n+962\right)
$$$$
\sum_{0 \leq j_{1}<j_{2}<j_{3}<j_{4} \leq n-1} g_{\alpha}^{j_{1} j_{2} j_{3} j_{4}} g_{\beta}^{j_{1} j_{2} j_{3} j_{4}}=\frac{n\left(n^{2}-1\right)\left(n^{2}-4\right)\left(n^{2}-9\right)}{57466158750}\left(148 n^{10}+12742 n^{8}+399559 n^{6}\right.
$$$$
\left.+9468311 n^{4}+149848193 n^{2}+1582095047\right)
$$$$
\sum_{0 \leq j_{1}<j_{2}<j_{3}<j_{4} \leq n-1} g_{\alpha}^{j_{1} j_{2} j_{3} j_{4}} g_{\gamma}^{j_{1} j_{2} j_{3} j_{4}}=\frac{2 n\left(n^{2}-1\right)\left(n^{2}-4\right)\left(n^{2}-9\right)}{1915538625}\left(13 n^{8}+1193 n^{6}+49371 n^{4}\right.
$$$$
\left.+1707707 n^{2}+27272116\right)
$$$$
\sum_{0 \leq j_{1}<j_{2}<j_{3}<j_{4} \leq n-1} g_{\alpha}^{j_{1} j_{2} j_{3} j_{4}} g_{\delta}^{j_{1} j_{2} j_{3} j_{4}}=\frac{n\left(n^{2}-1\right)\left(n^{2}-4\right)\left(n^{2}-9\right)}{638512875}\left(587 n^{6}+57813 n^{4}+1175013 n^{2}\right.
$$$$
+18724987)
$$$$
\sum_{0 \leq j_{1}<j_{2}<j_{3}<j_{4} \leq n-1} g_{\beta}^{j_{1} j_{2} j_{3} j_{4}} g_{\gamma}^{j_{1} j_{2} j_{3} j_{4}}=\frac{n\left(n^{2}-1\right)\left(n^{2}-4\right)\left(n^{2}-9\right)\left(n^{2}+39\right)}{212837625}\left(17 n^{6}+511 n^{4}\right.
$$$$
\left.+8323 n^{2}+71789\right)
$$ 


$$
\begin{aligned}
\sum_{0 \leq j_{1}<j_{2}<j_{3}<j_{4} \leq n-1} g_{\beta}^{j_{1} j_{2} j_{3} j_{4}} g_{\delta}^{j_{j} j_{2} j_{3} j_{4}}= & \frac{n(n-2)(n-3)\left(n^{2}-1\right)}{2554051500}\left(3003 n^{9}+10001 n^{8}+105060 n^{7}\right. \\
& +407500 n^{6}+2141874 n^{5}+7267638 n^{4}+28886340 n^{3} \\
& \left.+64096300 n^{2}+168447723 n+207636161\right), \\
\sum_{0 \leq j_{1}<j_{2}<j_{3}<j_{4} \leq n-1} g_{\gamma}^{j_{1} j_{2} j_{3} j_{4}} g_{\delta}^{j_{1} j_{2} j_{3} j_{4}}= & \frac{n(n-3)\left(n^{2}-1\right)\left(n^{2}-4\right)\left(n^{2}-3 n+26\right)}{467775}\left(3 n^{4}+14 n^{3}\right. \\
& \left.+104 n^{2}+370 n+1429\right) .
\end{aligned}
$$

Open Access. This article is distributed under the terms of the Creative Commons Attribution License (CC-BY 4.0), which permits any use, distribution and reproduction in any medium, provided the original author(s) and source are credited.

\section{References}

[1] M. Headrick, Entanglement Rényi entropies in holographic theories, Phys. Rev. D 82 (2010) 126010 [arXiv: 1006. 0047] [INSPIRE].

[2] P. Calabrese, J. Cardy and E. Tonni, Entanglement entropy of two disjoint intervals in conformal field theory II, J. Stat. Mech. 01 (2011) P01021 [arXiv:1011.5482] [INSPIRE].

[3] B. Chen and J.-J. Zhang, On short interval expansion of Rényi entropy, JHEP 11 (2013) 164 [arXiv: 1309.5453] [INSPIRE].

[4] M.A. Nielsen and I.L. Chuang, Quantum computation and quantum information, Cambridge University Press, Cambridge U.K. (2010).

[5] D. Petz, Quantum information theory and quantum statistics, Springer, Germany (2008).

[6] C.G. Callan Jr. and F. Wilczek, On geometric entropy, Phys. Lett. B 333 (1994) 55 [hep-th/9401072] [INSPIRE].

[7] P. Calabrese and J.L. Cardy, Entanglement entropy and quantum field theory, J. Stat. Mech. 06 (2004) P06002 [hep-th/0405152] [INSPIRE].

[8] P. Calabrese, J. Cardy and E. Tonni, Entanglement entropy of two disjoint intervals in conformal field theory, J. Stat. Mech. 11 (2009) P11001 [arXiv:0905.2069] [INSPIRE].

[9] P. Calabrese and J. Cardy, Entanglement entropy and conformal field theory, J. Phys. A 42 (2009) 504005 [arXiv: 0905.4013] [inSPIRE].

[10] I.R. Klebanov, S.S. Pufu, S. Sachdev and B.R. Safdi, Rényi entropies for free field theories, JHEP 04 (2012) 074 [arXiv:1111.6290] [INSPIRE].

[11] M.A. Rajabpour and F. Gliozzi, Entanglement entropy of two disjoint intervals from fusion algebra of twist fields, J. Stat. Mech. 02 (2012) P02016 [arXiv:1112.1225] [INSPIRE].

[12] J. Cardy, Some results on the mutual information of disjoint regions in higher dimensions, J. Phys. A 46 (2013) 285402 [arXiv: 1304.7985] [inSPIRE].

[13] A. Coser, L. Tagliacozzo and E. Tonni, On Rényi entropies of disjoint intervals in conformal field theory, J. Stat. Mech. 2014 (2014) P01008 [arXiv:1309.2189] [InSPIRE]. 
[14] S. Datta and J.R. David, Rényi entropies of free bosons on the torus and holography, arXiv: 1311.1218 [INSPIRE].

[15] J.M. Maldacena, The large- $N$ limit of superconformal field theories and supergravity, Adv. Theor. Math. Phys. 2 (1998) 231 [Int. J. Theor. Phys. 38 (1999) 1113] [hep-th/9711200] [INSPIRE].

[16] S.S. Gubser, I.R. Klebanov and A.M. Polyakov, Gauge theory correlators from noncritical string theory, Phys. Lett. B 428 (1998) 105 [hep-th/9802109] [INSPIRE].

[17] E. Witten, Anti-de Sitter space and holography, Adv. Theor. Math. Phys. 2 (1998) 253 [hep-th/9802150] [INSPIRE].

[18] S. Ryu and T. Takayanagi, Holographic derivation of entanglement entropy from AdS/CFT, Phys. Rev. Lett. 96 (2006) 181602 [hep-th/0603001] [INSPIRE].

[19] S. Ryu and T. Takayanagi, Aspects of holographic entanglement entropy, JHEP 08 (2006) 045 [hep-th/0605073] [INSPIRE].

[20] T. Nishioka, S. Ryu and T. Takayanagi, Holographic entanglement entropy: an overview, J. Phys. A 42 (2009) 504008 [arXiv:0905.0932] [inSPIRE].

[21] T. Takayanagi, Entanglement entropy from a holographic viewpoint, Class. Quant. Grav. 29 (2012) 153001 [arXiv:1204.2450] [INSPIRE].

[22] D.V. Fursaev, Proof of the holographic formula for entanglement entropy, JHEP 09 (2006) 018 [hep-th/0606184] [INSPIRE].

[23] D.V. Fursaev, Entanglement entropy in quantum gravity and the Plateau groblem, Phys. Rev. D 77 (2008) 124002 [arXiv:0711.1221] [INSPIRE].

[24] H. Casini, M. Huerta and R.C. Myers, Towards a derivation of holographic entanglement entropy, JHEP 05 (2011) 036 [arXiv:1102.0440] [INSPIRE].

[25] D.V. Fursaev, Entanglement Rényi entropies in conformal field theories and holography, JHEP 05 (2012) 080 [arXiv:1201.1702] [INSPIRE].

[26] A. Lewkowycz and J. Maldacena, Generalized gravitational entropy, JHEP 08 (2013) 090 [arXiv: 1304.4926] [INSPIRE].

[27] T. Faulkner, The entanglement Rényi entropies of disjoint intervals in AdS/CFT, arXiv:1303.7221 [INSPIRE].

[28] T. Hartman, Entanglement entropy at large central charge, arXiv:1303.6955 [INSPIRE].

[29] J.D. Brown and M. Henneaux, Central charges in the canonical realization of asymptotic symmetries: an example from three-dimensional gravity, Commun. Math. Phys. 104 (1986) 207 [INSPIRE].

[30] T. Barrella, X. Dong, S.A. Hartnoll and V.L. Martin, Holographic entanglement beyond classical gravity, JHEP 09 (2013) 109 [arXiv:1306.4682] [INSPIRE].

[31] T. Faulkner, A. Lewkowycz and J. Maldacena, Quantum corrections to holographic entanglement entropy, JHEP 11 (2013) 074 [arXiv: 1307.2892] [INSPIRE].

[32] A. Maloney and E. Witten, Quantum gravity partition functions in three dimensions, JHEP 02 (2010) 029 [arXiv:0712.0155] [INSPIRE]. 
[33] A. Campoleoni, S. Fredenhagen, S. Pfenninger and S. Theisen, Asymptotic symmetries of three-dimensional gravity coupled to higher-spin fields, JHEP 11 (2010) 007 [arXiv: 1008.4744] [INSPIRE].

[34] M. Henneaux and S.-J. Rey, Nonlinear $W_{\infty}$ as asymptotic symmetry of three-dimensional higher spin anti-de Sitter gravity, JHEP 12 (2010) 007 [arXiv:1008.4579] [INSPIRE].

[35] E. Perlmutter, Comments on Rényi entropy in $A d S_{3} / C F T_{2}$, arXiv:1312.5740 [INSPIRE].

[36] B. Chen, J. Long and Y.-N. Wang, Black holes in truncated higher spin AdS $S_{3}$ gravity, JHEP 12 (2012) 052 [arXiv: 1209.6185] [INSPIRE].

[37] X. Yin, Partition functions of three-dimensional pure gravity, Commun. Num. Theor. Phys. 2 (2008) 285 [arXiv:0710.2129] [INSPIRE].

[38] S. Giombi, A. Maloney and X. Yin, One-loop partition functions of $3 D$ gravity, JHEP 08 (2008) 007 [arXiv:0804.1773] [INSPIRE].

[39] M.R. Gaberdiel, R. Gopakumar, T. Hartman and S. Raju, Partition functions of holographic minimal models, JHEP 08 (2011) 077 [arXiv:1106.1897] [INSPIRE].

[40] M.R. Gaberdiel, R. Gopakumar and A. Saha, Quantum $W$-symmetry in $A d S_{3}$, JHEP 02 (2011) 004 [arXiv: 1009.6087] [INSPIRE].

[41] M.R. Gaberdiel and R. Gopakumar, Minimal model holography, J. Phys. A 46 (2013) 214002 [arXiv:1207.6697] [InSPIRE].

[42] B. Chen, F.-Y. Song and J.-J. Zhang, Holographic Rényi entropy in $A d S_{3} / L C F T_{2}$ correspondence, arXiv:1401.0261 [INSPIRE].

[43] M. Ammon, A. Castro and N. Iqbal, Wilson lines and entanglement entropy in higher spin gravity, JHEP 10 (2013) 110 [arXiv: 1306.4338] [INSPIRE].

[44] J. de Boer and J.I. Jottar, Entanglement entropy and higher spin holography in $A d S_{3}$, arXiv:1306.4347 [INSPIRE]. 